Research Article

\title{
Synchronization Analysis for Stochastic T-S Fuzzy Complex Networks with Markovian Jumping Parameters and Mixed Time-Varying Delays via Impulsive Control
}

\author{
M. Syed Ali, ${ }^{1}$ M. Usha, ${ }^{1}$ Quanxin Zhu ${ }^{1 D},{ }^{2}$ and Saravanan Shanmugam ${ }^{3,4}$ \\ ${ }^{1}$ Department of Mathematics, Thiruvalluvar University, Vellore 632115, Tamil Nadu, India \\ ${ }^{2}$ MOE-LCSM, School of Mathematics and Statistics, Hunan Normal University, Changsha 410081, China \\ ${ }^{3}$ School of Mechanical Engineering, Pusan National University, Busan 46241, Republic of Korea \\ ${ }^{4}$ School of Information Science and Engineering, Chengdu University, Chengdu 610106, China \\ Correspondence should be addressed to Quanxin Zhu; zqx22@126.com
}

Received 27 October 2019; Revised 29 May 2020; Accepted 20 June 2020; Published 28 July 2020

Academic Editor: Jose de Jesus Rubio

Copyright (C) 2020 M. Syed Ali et al. This is an open access article distributed under the Creative Commons Attribution License, which permits unrestricted use, distribution, and reproduction in any medium, provided the original work is properly cited.

In this paper, we propose and explore the synchronization examination for fuzzy stochastic complex networks' Markovian jumping parameters portrayed by Takagi-Sugeno (T-S) fuzzy model with mixed time-varying coupling delays via impulsive control. The hybrid coupling includes time-varying discrete and distributed delays. Based on appropriate Lyapunov-Krasovskii functional (LKF) approach, Newton-Leibniz formula, and Jensen's inequality, the stochastic examination systems and Kronecker product to create delay-dependent synchronization criteria that guarantee stochastically synchronous of the proposed T-S fuzzy stochastic complex networks with mixed timevarying delays. Adequate conditions for the synchronization criteria for the frameworks are established in terms of linear matrix inequalities (LMIs). At long last, numerical examples and simulations are given to demonstrate the correctness of the hypothetical outcomes.

\section{Introduction}

Complex systems [1-5] have obtained significant consideration in recent years. They are generally utilized in different areas of engineering, biology, material science, mathematics, human science monetary science, and shale-gas extraction [6-9]. It is commonly realized that complex systems consist of a sizeable amount of nodes and edges, and every node and edge have its very own correspondent interpretation; along these lines, numerous frameworks as a general rule can be described by complex frameworks, for example, the networking net, traffic systems, neural systems, epidemic spreading, scientific citations, WWW, informal organizations, power matrices, global economic markets, etc [2-12]. Different logical models were expected to portray diverse complex systems, small world systems [13] and scale-free systems [14], and so forth. Complex systems normally show unpredictable and entrancing dynamical lead including synchronization [15], consensus [16], running, and so forth.
The synchronization and control of complex structures have been generally inspected in different areas of planning as well as innovation because of its numerous hidden reality purposes. Time delays may cause unfortunate powerful system practices, for example, wavering and instability [17]. Synchronization of complex networks has found extensive applications in fields such as data ferrying with unmanned aerial vehicles, image encryption, secure communication, biological systems, chemical reaction, nonlinear oscillation, chaos generator design, and swarm intelligence $[18,19]$. As a rule, the fundamental thought of drive-response synchronization is to construct the controlled slave system to reach the same behavior with the autonomous drive system. The synchronization phenomena are common and essential in actual-world networks, along with synchronization at the net, synchronization transfer of digital or analog signals in communique networks, and synchronization related to organic neural networks. Along these lines, various scientists have looked at the synchronization of complex frameworks 
with delays and a lot of significant comments have been acquired $[20,21]$.

In practice, a real system is often affected by external random perturbations, i.e., the system has stochastic effects which can also lead to complex dynamic behaviours together with oscillation, divergence, chaos, instability, or different terrible performance. On the other hand, within the real world, complex networks are often challenging to environmental disturbances, and therefore, the stochastic modeling issue has been of vital importance. Especially, the framework models are commonly upset by outer disturbances which can be treated as stochastic problems of the structure. Array of networks scalar Wiener processes, is chatted on [22], which internal that each node is influenced by the noise.

In recent years, Markovian jump complex networks (MJCNs) have received increasing attention inside the area of control. Markovian jump systems may be defined through a set of linear systems that switch transfer from one to another at various times with a certain transition rate [23]. Dynamic structures can be exhibited by an uncommon class of hybrid system, for a model Markovian jump complex dynamical systems. Markovian chains are utilized for the jumping of finite nodes of the complex dynamical systems which may jump from one to another at various times. The frameworks with Markovian jump parameters, which move by ceaseless-time Markov chains, have been extensively used to show various genuine frameworks, where they may rehearse unexpected modification in their network and parameters. The jump structures have the benefit of showing the powerful frameworks branch of knowledge to unexpected variety in their systems, for example, part disappointments or fixes, abrupt ecological unsettling influence, and working at various purposes of a nonlinear systems [24-27]. In addition, in Markovian jump complex networks, the jumps in operation modes are governed by the Markov process or Markov chain.

Recently, many control techniques have been introduced to structure valuable controllers, such as adaptive, intermittent, impulsive, pinning, sampling data, sliding mode, and so on. Impulsive controller is a marvel that has been taken into discussion when modeling the complex networks. Impulsive frameworks normally are an extensive variation of developmental procedures wherein components, all of a sudden, change at explicit depictions of time [28-30]. Scientifically, these impulsive frameworks are generally portrayed by the relating impulsive differential conditions, which are the model of impulsive control frameworks [31-37]. We all know that various organic wonders incorporating blasting mood models in science and ideal control models in financial aspects show motivation impacts.

In the previous decades, Takagi-Sugeno (T-S) fuzzy version, primarily based on the dead control technique, was generally used to appropriate numerous complex nonlinear systems, and as of late it has turned out to be dynamic because of its wide designing applications $[38,39]$. T-S fuzzy frameworks are nonlinear frameworks depicted by a set of IF-THEN standards. The T-S fuzzy model is a powerful one to break down, and its nonlinear elements are found everywhere in sign handling, synthetic procedures, and mechanical technology frameworks [40-42]. Furthermore, the fuzzy logic control procedure, as of late, has turned into a functioning exploration subject due to its potential applications in various fields [43-51]. On the other hand, to the creators' information, up until this point, the issue of synchronization considering T-S fuzzy complicated systems by Markovian jumping as well as stochastic coupling postponement has not been tended to in the writing.

Motivated by the examinations mentioned above, this article intends to explore about the synchronization investigation for Markovian jumping parameters with stochastic discrete and distributed time-varying delays by means of impulsive control. We constructed a novel Lyapunov-Krasovskii functional (LKF) and derived delay-dependent synchronization criteria for drive-response Markovian jump complex systems with mixed time varying delays in terms of linear matrix inequalities (LMIs). We employed the properties of Kronecker product approach and stochastic assessment techniques to derive the proposed results. All the derived conditions arrived are communicated as LMIs whose feasibility could be successfully investigated by utilizing mathematically effective MATLAB LMI Control toolbox. Finally, a numerical model is given to show the adequacy of the proposed outcomes.

Notations 1. Throughout this article, $\mathbb{R}^{+}$and $\mathbb{R}^{n}$ indicate, individually, the arrangement of nonnegative real numbers and the $n$-dimensional Euclidean space. The superscript " $T$ " represents matrix transposition and characters $\mathscr{B}>\mathscr{A}$ (individually, $\mathscr{B} \leq \mathscr{A}$ ) indicate $\mathscr{A}-\mathscr{B}$ is semidefinite (individually, positive definite). $\mathrm{N}^{+}$represents the set of positive integers and diag $\{\ldots\}$ stands for block-diagonal matrix. The asterisk " $*$ " in a symmetric matrix is utilized to mean term that is induced by symmetry. $I$ and 0 represent the identity matrix and a zero matrix, respectively. The Kronecker product of grids $\mathbb{Q} \in \mathbb{R}^{m \times n}$ as well as $\mathscr{R} \in \mathbb{R}^{p \times q}$ in $\mathbb{R}^{m p \times n q}$ is signified as $\mathbb{Q} \otimes \mathscr{R} . \mathbb{E}\{\cdot\}$ represents the mathematical expectation administrator. $\|\cdot\|$ indicates the Euclidean standard of a vector and its induced standard of a matrix. Let $\left(\Omega, \mathscr{F},\left\{\mathscr{F}_{t}\right\}_{t \geq 0}, \mathscr{P}\right)$ be a complete probability space which relates to an increasing family $\left\{\mathscr{F}_{t}\right\}_{t \geq 0}$ of $\sigma$ algebras $\left\{\mathscr{F}_{t}\right\}_{t \geq 0} \subset \mathscr{F}$, where $\Omega$ denotes the sample space, $\mathscr{F}$ represents $\sigma$ algebra of subsets on the sample space, and $\mathscr{P}$ is the probability measure defined on $\mathscr{F}$. Let $\{9(t)(t \geq 0)\}$ be a right continuous Markovian chain on the probability space $\left(\Omega, \mathscr{F},\left\{\mathscr{F}_{t}\right\}_{t \geq 0}, \mathscr{P}\right)$ taking values in the finite space $\delta=$ $\{1,2, \ldots, n\}$ with the initial model $\vartheta(0)=\vartheta_{0}$. Along generator $\Theta=\left(\theta_{\kappa j}\right)_{n \times n}$, let $(\kappa, j \in \mathcal{S})$ be the transition rate matrix with transition probability

$$
\operatorname{Pr}\{\vartheta(t+\Delta)=j \mid \vartheta(t)=\kappa)\}= \begin{cases}\theta_{\kappa j} \Delta+o(\Delta), & \text { if } \kappa \neq j, \\ 1+\theta_{\kappa \kappa} \Delta+o(\Delta), & \text { if } \kappa=j,\end{cases}
$$

where $\Delta>0$, and $\theta_{\kappa j} \geq 0$, is the progress rate mode $\kappa$ to $j$ if $\kappa \neq j$ and 


$$
\theta_{\kappa \kappa}=-\sum_{j=1, j \neq \kappa} \theta_{\kappa j} .
$$

Besides, the transition probabilities matrix $\Theta$ is indicated as follows:

$$
\Theta=\left[\begin{array}{cccc}
\theta_{11} & \theta_{12} & \cdots & \theta_{1 n} \\
\theta_{21} & \theta_{22} & \cdots & \theta_{2 n} \\
\vdots & \vdots & \ddots & \vdots \\
\theta_{n 1} & \theta_{n 2} & \cdots & \theta_{n n}
\end{array}\right] .
$$

identical nodes with drive-response Markovian jumping parameters by a T-S fuzzy model. The system dynamics can be captured by a set of fuzzy rules which can characterize local correlations in the state space. The subsequent rules are as follows.

Drive system rule l:

$$
\begin{aligned}
& \text { IF }\left\{\Lambda_{1}(t) \text { is } \chi_{1}^{l}\right\} \text { and }\left\{\Lambda_{2}(t) \text { is } \chi_{2}^{l}\right\} \text { and } \ldots \text { and } \\
& \left\{\Lambda_{g}(t) \text { is } \chi_{g}^{l}\right\}, \\
& \text { THEN }
\end{aligned}
$$

\section{Problem Description and Preliminaries}

In this paper, we consider the following stochastic impulsive complex networks with coupling delays consisting of $N$

$$
\left\{\begin{array}{l}
\mathrm{d} x_{i}(t)=\left[\mathscr{A}_{l}(\vartheta(t)) x_{i}(t)+\mathscr{B}_{l}(\vartheta(t)) f\left(x_{i}(t)\right)+\mathscr{C}_{l}(\vartheta(t)) g\left(x_{i}(t-\tau(t))\right)+\mathscr{D}_{l}(\vartheta(t)) \int_{t-\varrho(t)}^{t} h\left(x_{i}(s)\right) \mathrm{d} s\right. \\
\left.\quad+c_{1} \sum_{j=1}^{N} \mathscr{G}_{1 l i j} \Gamma_{1}(\vartheta(t)) x_{i}(t)+c_{2} \sum_{j=1}^{N} \mathscr{G}_{2 l i j} \Gamma_{2}(\vartheta(t)) x_{i}(t-\tau(t))+c_{3} \sum_{j=1}^{N} \mathscr{G}_{3 l i j} \Gamma_{3}(\vartheta(t)) \int_{t-\varrho(t)}^{t} x_{j}(s) \mathrm{d} s+\mathscr{I}_{i}(t)\right] \mathrm{d} t \\
\quad+\rho_{l i}\left(t, x_{i}(t), x_{i}(t-\tau(t)), \int_{t-\varrho(t)}^{t} x_{i}(s) \mathrm{d} s\right) \mathrm{d} \omega(t), \quad t \neq t_{k}, t \geq t_{0}, \\
x_{i}\left(t_{k}\right)=\mathscr{J}_{l}(\vartheta(t)) x_{i}\left(t_{k}^{-}\right), \quad i=1,2, \ldots, N, t=t_{k}, k \in N^{+} \\
x_{i}(t)=\phi_{i}(t), \quad t \in[-\max \{\tau, \varrho\}, 0] .
\end{array}\right.
$$

Response system rule 1:

THEN

$$
\begin{aligned}
& \text { IF }\left\{\Lambda_{1}(t) \text { is } \chi_{1}^{l}\right\} \text { and }\left\{\Lambda_{2}(t) \text { is } \chi_{2}^{l}\right\} \text { and } \ldots \text { and } \\
& \left\{\Lambda_{g}(t) \text { is } \chi_{g}^{l}\right\},
\end{aligned}
$$

$$
\left\{\begin{array}{l}
\mathrm{d} \hat{y}_{i}(t)=\left[\mathscr{A}_{l}(\vartheta(t)) \hat{y}_{i}(t)+\mathscr{B}_{l}(\vartheta(t)) f\left(\hat{y}_{i}(t)\right)+\mathscr{C}_{l}(\vartheta(t)) g\left(\hat{y}_{i}(t-\tau(t))\right)+\mathscr{D}_{l}(\vartheta(t)) \int_{t-\varrho(t)}^{t} h\left(\hat{y}_{i}(s)\right) \mathrm{d} s\right. \\
\quad+c_{1} \sum_{j=1}^{N} \mathscr{G}_{1 l i j} \Gamma_{1}(\vartheta(t)) \hat{y}_{i}\left(\hat{y}_{i}(s)\right) \mathrm{d} s+c_{2} \sum_{j=1}^{N} \mathscr{G}_{2 l i j} \Gamma_{2}(\vartheta(t)) \hat{y}_{i}(t-\tau(t)) \\
\left.\quad+c_{3} \sum_{j=1}^{N} \mathscr{G}_{3 l i j} \Gamma_{3}(\vartheta(t)) \int_{t-\varrho(t)}^{t} \hat{y}_{j}(s) \mathrm{d} s+\mathscr{F}_{i}(t)\right] \mathrm{d} t \\
\quad+\rho_{l i}\left(t, \hat{y}_{i}(t), \hat{y}_{i}(t-\tau(t)), \int_{t-\varrho(t)}^{t} \hat{y}_{i}(s) \mathrm{d} s\right) \mathrm{d} \omega(t), \quad t \neq t_{k}, t \geq t_{0}, \\
\hat{y}_{i}\left(t_{k}\right)=\mathscr{F}_{l}(\vartheta(t)) \hat{y}_{i}\left(t_{k}^{-}\right), \quad i=1,2, \ldots, N, \quad t=t_{k}, k \in N^{+} \\
\hat{y}_{i}(t)=\varphi_{i}(t), \quad t \in[-\max \{\tau, \varrho\}, 0],
\end{array}\right.
$$

where $l \in S=(1,2, \ldots, v) . v$ is the number of fuzzy IFTHEN rules. $\chi_{1}^{l}, \ldots, \chi_{q}^{l}$ are fuzzy sets that are characterized by the membership functions; $\Lambda(t)=\left[\Lambda_{1}(t), \ldots, \Lambda_{g}(t)\right]^{T}$ are premise variables; $x_{i}(t)=\left[x_{i 1}(t), x_{i 2}(t), \ldots\right.$, $\left.x_{\text {in }}(t)\right]^{T} \in \mathbb{R}^{n}$ is the state vector of drive system (4) associated with $i$ th node at time $t ; \hat{y}_{i}(t)=\left[\hat{y}_{i 1}(t), \hat{y}_{i 2}(t), \ldots\right.$, $\left.\hat{y}_{i n}(t)\right]^{T} \in \mathbb{R}^{n}$ is the state vector of response system (5) associated with $i$ th node at time $t ; \mathscr{A}_{l}(\vartheta(t)), \mathscr{B}_{l}(\vartheta(t))$, $\mathscr{C}_{l}\left(\vartheta((t))\right.$, and $\mathscr{D}_{l}(\vartheta(t))$ are known constant matrices with appropriate dimensions; $c_{1}>0, c_{2}>0$, and $c_{3}>0$ are constants denoting the coupling strengths; $f\left(x_{i}(t)\right)=$ $\operatorname{col}\left[f_{1}\left(x_{i 1}(t)\right), \ldots, f_{n}\left(x_{i n}(t)\right)\right]^{T}, \quad g\left(x_{i}(t)\right)=\operatorname{col}\left[g_{1}\left(x_{i 1}\right.\right.$ $\left.(t)), \ldots, g_{n_{T}}\left(x_{i n}(t)\right)\right]^{T^{n}}$ and $h\left(x_{i}(t)\right)=\operatorname{col}\left[h_{1}\left(x_{i 1}(t)\right), \ldots\right.$, $\left.h_{n}\left(x_{i n}(t)\right)\right]^{T}, \quad f\left(\hat{y}_{i}(t)\right)=\operatorname{col}\left[f_{1}\left(\hat{y}_{i 1}(t)\right), \ldots, f_{n}\left(\hat{y}_{i n}(t)\right)\right]^{T}$, 
and $\quad g\left(\hat{y}_{i}(t)\right)=\operatorname{col}\left[g_{1}\left(\hat{y}_{i 1}(t)\right), \ldots, g_{n}\left(\hat{y}_{i n}(t)\right)\right]^{T} \quad$ and $h\left(\hat{y}_{i}(t)\right)=\operatorname{col}\left[h_{1}\left(\hat{y}_{i 1}(t)\right), \ldots, h_{n}\left(\hat{y}_{i n}(t)\right)\right]^{T}$ are unknown but sector-bounded nonlinear functions of drive and response framework (4) and (5) individually, where $0<\tau(t)$ including $0<\varrho(t)$ signifies discrete and distributed timevarying delays, which are accepted to fulfilling

$$
\begin{aligned}
& 0 \leq \tau(t) \leq \tau, \\
& \dot{\tau}(t) \leq \mu<1, \\
& 0 \leq \varrho(t) \leq \varrho,
\end{aligned}
$$

respectively, where $\tau$, $\varrho$, and $\mu$ are consistent and are selfdetermining of the Markovian process $\{\vartheta(t)\}_{t \geq 0}$; furthermore, $\rho_{l i}(\cdot, \cdot, \cdot, \cdot): \mathbb{R} \times \mathbb{R}^{n} \times \mathbb{R}^{n} \longrightarrow \mathbb{R}^{n}$ is the annoyance force function vector; $\omega(t)=\left[\omega_{1}(t), \omega_{2}(t), \ldots, \omega_{n}(t)\right]^{T}$ is an $n$-dimensional Brownian motion determined on a complete probability space $\left(\Omega, \mathscr{F},\left\{\mathscr{F}_{t}\right\}_{t \geq 0}, \mathscr{P}\right)$ satisfying

$$
\begin{aligned}
\mathbb{E}\{\mathrm{d} \omega(t)\} & =0, \\
\mathbb{E}\left\{[\mathrm{d} \omega(t)]^{2}\right\} & =\mathrm{d} t,
\end{aligned}
$$

where $\mathscr{I}_{i}(t)$ is the constant external input. $\mathscr{I}_{l}(\vartheta(t))$ denotes the impulse gain matrix at the moment of time $t_{k}$. The discrete set $\left\{t_{k}\right\}$ satisfies $0=t_{0}<t_{1}<\cdots<t_{k}<\cdots$ $\lim _{k \rightarrow \infty} t_{k}=+\infty . x_{i}\left(t_{k}^{-}\right), \hat{y}_{i}\left(t_{k}^{-}\right)$and $x_{i}\left(t_{k}^{+}\right), \hat{y}_{i}\left(t_{k}^{+}\right)$denote the left-hand and right-hand limits at $t_{k}$ of drive and response system, respectively. $\phi_{i}(t)$ and $\varphi_{i}(t)$ are continuously vector-valued initial condition defined on $[-\max \{\tau, \varrho\}, 0]$ of the drive and response system. We assume that $x_{i}\left(t_{k}\right)$ and $\hat{y}_{i}\left(t_{k}\right)$ are right continuous, i.e., $x_{i}\left(t_{k}^{+}\right)=x_{i}\left(t_{k}\right)$ and $\hat{y}_{i}\left(t_{k}^{+}\right)=\hat{y}_{i}\left(t_{k}\right) . \quad \Gamma_{a}(\vartheta(t))=\operatorname{diag}\left\{q_{a 1}(\vartheta(t)), q_{a 2}(\vartheta(t)), \ldots\right.$, $\left.q_{a n}(\vartheta(t))\right\}(a=1,2,3)$ are constant diagonal inner-coupling matrices among the subsystems; $\mathscr{G}_{1 l}=\left(\mathscr{G}_{1 l i j}\right)_{N \times N}$, $\mathscr{G}_{2 l}=\left(\mathscr{G}_{2 l i j}\right)_{N \times N}$, and $\mathscr{G}_{3 l}=\left(\mathscr{G}_{3 l i j}\right)_{N \times N}$ continue the external combination arrangement matrices characterizing the topological structure of the complex systems in which $\mathscr{G}_{\text {qlij }}(q=1,2,3)$ is characterized as follows: if there is association within node $i$ and $j(i \neq j)$, at that point $\mathscr{G}_{q l i j}=\mathscr{G}_{q l j i}=1$; otherwise, $\mathscr{G}_{q l i j}=\mathscr{G}_{q l j i}=0,(i \neq j)$, and the diagonal components of matrices $\mathscr{G}_{q l}$ are characterized by

$$
\left\{\begin{array}{l}
\mathscr{G}_{1 l i i}=\sum_{j=1, j \neq i}^{N} \mathscr{G}_{1 l i j}=-\sum_{j=1, j \neq i}^{N} \mathscr{G}_{1 l i j}, \\
\mathscr{G}_{2 l i i}=\sum_{j=1, j \neq i}^{N} \mathscr{G}_{2 l i j}=-\sum_{j=1, j \neq i}^{N} \mathscr{G}_{2 l i j}, \\
\mathscr{G}_{3 l i i}=\sum_{j=1, j \neq i}^{N} \mathscr{G}_{3 l i j}=-\sum_{j=1, j \neq i}^{N} \mathscr{G}_{3 l i j}, \quad l=1,2, \ldots, v, i, j=1,2, \ldots, N .
\end{array}\right.
$$

Utilizing the singleton fuzzifier, product fuzzy inference, and weighted average defuzzifier [52], the yield of above fuzzy drive framework is gathered as follows:

Drive system rule 1:
IF $\left\{\Lambda_{1}(t)\right.$ is $\left.\chi_{1}^{l}\right\}$ and $\left\{\Lambda_{2}(t)\right.$ is $\left.\chi_{2}^{l}\right\}$ and $\ldots$ and $\left\{\Lambda_{g}(t)\right.$ is $\left.\chi_{g}^{l}\right\}$,

THEN

$$
\left\{\begin{array}{l}
\mathrm{d} x_{i}(t)=\sum_{l=1}^{v} \delta_{l}(\Lambda(t))\left\{\left[\mathscr{A}_{l}(\vartheta(t)) x_{i}(t)+\mathscr{B}_{l}(\vartheta(t)) f\left(x_{i}(t)\right)+\mathscr{C}_{l}(\vartheta(t)) g\left(x_{i}(t-\tau(t))\right)\right.\right. \\
\quad+\mathscr{D}_{l}(\vartheta(t)) \int_{t-\varrho(t)}^{t} h\left(x_{i}(s)\right) \mathrm{d} s+c_{1} \sum_{j=1}^{N} \mathscr{G}_{1 l i j} \Gamma_{1}(\vartheta(t)) x_{i}(t)+\mathscr{I}_{i}(t)+c_{2} \sum_{j=1}^{N} \mathscr{G}_{2 l i j} \Gamma_{2}(\vartheta(t)) x_{i}(t-\tau(t)) \\
\left.\left.\left.\quad+c_{3} \sum_{j=1}^{N} \mathscr{G}_{3 l i j} \Gamma_{3}(\vartheta(t)) \int_{t-\varrho(t)}^{t} x_{j}(s) \mathrm{d} s\right]\right\} \mathrm{~d} t+\rho_{l i}\left(t, x_{i}(t), x_{i}(t-\tau(t)), \int_{t-\varrho(t)}^{t} x_{i}(s) \mathrm{d} s\right) \mathrm{d} \omega(t)\right\}, \quad t \neq t_{k}, t \geq t_{0}, x_{i}\left(t_{k}\right), \\
x_{i}\left(t_{k}\right)=\sum_{l=1}^{v} \delta_{l}(\Lambda(t)) \mathscr{J}_{l}(\vartheta(t)) x_{i}\left(t_{k}^{-}\right), \quad i=1,2, \ldots, N, t=t_{k}, k \in N^{+}, \\
x_{i}(t)=\phi_{i}(t), \quad t \in[-\max \{\tau, \varrho\}, 0],
\end{array}\right.
$$


where

$$
\delta_{l}(\Lambda(t))=\frac{\prod_{j=1}^{v} \varpi_{l j}\left[\Lambda_{j}(t)\right]}{\sum_{l=1}^{v} \prod_{j=1}^{g} \Phi_{l j}\left[\Lambda_{j}(t)\right]}, \quad l=1,2, \ldots, v,
$$

in which $\varpi_{l j}\left[\Lambda_{j}(t)\right]$ is the grade of membership of $\Lambda_{j}(t)$ in $\chi_{g}^{l}$ and $\Lambda(t)=\left(\Lambda_{1}(t), \ldots, \Lambda_{g}(t)\right)$. Then, it can be seen that for $l=1,2, \ldots, v$ and for all $t$, we assume

$$
\delta_{l}(\Lambda(t)) \geq 0
$$

for all $t \in \mathscr{R}^{+}$, where $\delta_{l}(\Lambda(t)) \geq 0$ can be regarded as the normalized weight of the IF-THEN rules.

Compared to the fuzzy drive framework (4), the response framework is represented by the subsequent rules.

Response system rule 1:

IF $\left\{\Lambda_{1}(t)\right.$ is $\left.\chi_{1}^{l}\right\}$ and $\left\{\Lambda_{2}(t)\right.$ is $\left.\chi_{2}^{l}\right\}$ and $\ldots$ and $\left\{\Lambda_{g}(t)\right.$ is $\left.\chi_{g}^{l}\right\}$,

THEN

$$
\sum_{l=1}^{v} \delta_{l}(\Lambda(t))=1, l=1,2, \ldots, v
$$

From the drive system (9) and response system (12), the error system can be expressed as follows:

$$
\left\{\begin{array}{l}
\mathrm{d} e_{i}(t)=\sum_{l=1}^{v} \delta_{l}(\Lambda(t))\left\{\left[\mathscr{A}_{l}(\vartheta(t)) e_{i}(t)+\mathscr{B}_{l}(\vartheta(t)) f\left(e_{i}(t)\right)+\mathscr{C}_{l}(\vartheta(t)) g\left(e_{i}(t-\tau(t))\right)+\mathscr{D}_{l}(\vartheta(t)) \int_{t-\varrho(t)}^{t} h\left(e_{i}(s)\right) \mathrm{d} s\right.\right. \\
\left.\quad+c_{1} \sum_{j=1}^{N} \mathscr{G}_{1 l i j} \Gamma_{1}(\vartheta(t)) e_{i}(t)+c_{2} \sum_{j=1}^{N} \mathscr{G}_{2 l i j} \Gamma_{2}(\vartheta(t)) e_{i}(t-\tau(t))+c_{3} \sum_{j=1}^{N} \mathscr{G}_{3 l i j} \Gamma_{3}(\vartheta(t)) \int_{t-\varrho(t)}^{t} e_{j}(s) \mathrm{d} s\right] \mathrm{d} t \\
\left.\quad+\rho_{l i}\left(t, e_{i}(t), e_{i}(t-\tau(t)), \int_{t-\varrho(t)}^{t} e_{i}(s) \mathrm{d} s\right) \mathrm{d} \omega(t)\right\}, \quad t \neq t_{k}, t \geq t_{0}, \\
e_{i}\left(t_{k}\right)=\sum_{l=1}^{v} \delta_{l}(\Lambda(t)) \mathscr{F}_{l}(\vartheta(t)) e_{i}\left(t_{k}^{-}\right), \quad i=1,2, \ldots, N, \quad t=t_{k}, k \in N^{+}, \\
e_{i}(t)=\phi_{i}(t)-\varphi_{i}(t), \quad t \in[-\max \{\tau, \varrho\}, 0],
\end{array}\right.
$$

where $f\left(e_{i}(t)\right)=f\left(x_{i}(t)\right)-f\left(\hat{y}_{i}(t)\right),{ }_{t} g\left(e_{i}(t-\tau(t))\right)=$ $\begin{array}{cr}g\left(x_{i}(t-\tau(t))\right)-g\left(\hat{y}_{i}(t-\tau(t))\right), & \int_{t-\varrho(t)}^{t} h\left(e_{i}(s)\right) \mathrm{d} s= \\ \int_{t-\varrho(t)}^{t} h\left(x_{i}(s)\right) \mathrm{d} s-\int_{t-\rho(t)}^{t} h\left(\hat{y}_{i}(s)\right) \mathrm{d} s, & e_{i}(t-\tau(t))=\end{array}$ $x_{i}(t-\tau(t))-\hat{y}_{i}(t-\tau(t)), \rho_{l i}\left(t, e_{i}(t), e_{i}(t-\tau(t)), \int_{t-\rho(t)}^{t}\right.$ $\left.\left.e_{i}(s) \mathrm{d} s\right)=\rho_{l i}\left(t, x_{i}(t)\right)_{t} x_{i} \quad(t-\tau(t)) \int_{t-\varrho(t)}^{t} x_{i} \quad(s) \mathrm{d} s\right)-\rho_{l i}(t$, $\left.\hat{y}_{i}(t), \hat{y}_{i}(t-\tau(t)), \int_{t-\rho(t)}^{t} \hat{y}_{i}(s) \mathrm{d} s\right)$.

For comfort, every conceivable estimation of $\vartheta(t)$ is meant by $\kappa, \kappa \in \mathcal{S}$ in the sequel. At the point, we have

$$
\begin{aligned}
\mathscr{A}_{l \kappa} & =\mathscr{A}_{l}(\vartheta(t)), \\
\mathscr{B}_{l \kappa} & =\mathscr{B}_{l}(\vartheta(t)), \\
\mathscr{C}_{l \kappa} & =\mathscr{C}_{l}(\vartheta(t)), \\
\mathscr{D}_{l \kappa} & =\mathscr{D}_{l}(\vartheta(t)), \\
\Gamma_{1}(\vartheta(t)) & =\Gamma_{1 \kappa}, \\
\Gamma_{2}((\vartheta(t)) & =\Gamma_{2 \kappa},
\end{aligned}
$$


where $\mathscr{A}_{l \kappa}, \mathscr{B}_{l \kappa}, \mathscr{C}_{l \kappa}$, and $\mathscr{D}_{l \kappa}$ for any $\kappa \in \mathcal{S}$ are known constant frameworks of suitable measurements.

$$
\left\{\begin{aligned}
& \mathrm{d} e_{i}(t)=\sum_{l=1}^{v} \delta_{l}(\Lambda(t))\left\{\left[\mathscr{A}_{l \kappa} e_{i}(t)+\mathscr{B}_{l \kappa} f\left(e_{i}(t)\right)+\mathscr{C}_{l \kappa} g\left(e_{i}(t-\tau(t))\right)+\mathscr{D}_{l \kappa} \int_{t-\varrho(t)}^{t} h\left(e_{i}(s)\right) \mathrm{d} s+c_{1} \sum_{j=1}^{N} \mathscr{G}_{1 l i j} \Gamma_{1 \kappa} e_{i}(t)\right.\right. \\
&\left.\left.\quad+c_{2} \sum_{j=1}^{N} \mathscr{G}_{2 l i j} \Gamma_{2 \kappa} e_{i}(t-\tau(t))+c_{3} \sum_{j=1}^{N} \mathscr{G}_{3 l i j} \Gamma_{3 \kappa} \int_{t-\varrho(t)}^{t} e_{j}(s) \mathrm{d} s\right] \mathrm{~d} t+\rho_{l i}\left(t, e_{i}(t), e_{i}(t-\tau(t)), \int_{t-\varrho(t)}^{t} e_{i}(s) \mathrm{d} s\right) \mathrm{d} \omega(t)\right\}, \quad t \neq t_{k}, t \geq t_{0} \\
& e_{i}\left(t_{k}\right)=\sum_{l=1}^{v} \delta_{l}(\Lambda(t)) \mathscr{J}_{l \kappa} e_{i}\left(t_{k}^{-}\right), \quad i=1,2, \ldots N, \quad t=t_{k}, k \in N^{+}, \\
& e_{i}(t)=\phi_{i}(t)-\varphi_{i}(t), \quad t \in[-\max \{\tau, \varrho\}, 0] .
\end{aligned}\right.
$$

For simplicity, let

$$
\begin{aligned}
& e(t)=\left(e_{1}^{T}(t), e_{2}^{T}(t), \ldots, e_{N}^{T}(t)\right)^{T}, \\
& F(e(t))=\left(f^{T}\left(e_{1}(t)\right), f^{T}\left(e_{2}(t)\right), \ldots, f^{T}\left(e_{N}(t)\right)\right)^{T}, \\
& G(e(t-\tau(t)))=\left(g^{T}\left(e_{1}(t-\tau(t))\right), g^{T}\left(e_{2}(t-\tau(t))\right), \ldots, g^{T}\left(e_{N}(t-\tau(t))\right)\right)^{T}, \\
& H(e(t))=\left(h^{T}\left(e_{1}(t)\right), h^{T}\left(e_{2}(t)\right), \ldots, h^{T}\left(e_{N}(t)\right)\right)^{T},
\end{aligned}
$$

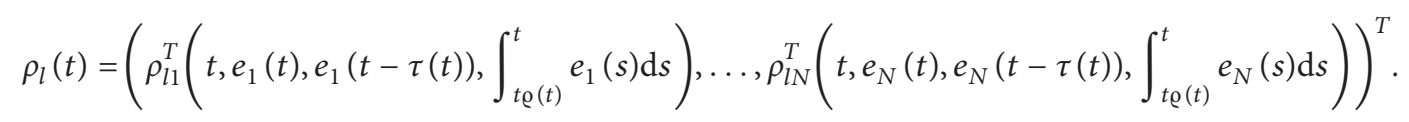

By employing the Kronecker product “ $\otimes$ ” of matrices, system (10) can be written in a compact structure as follows:

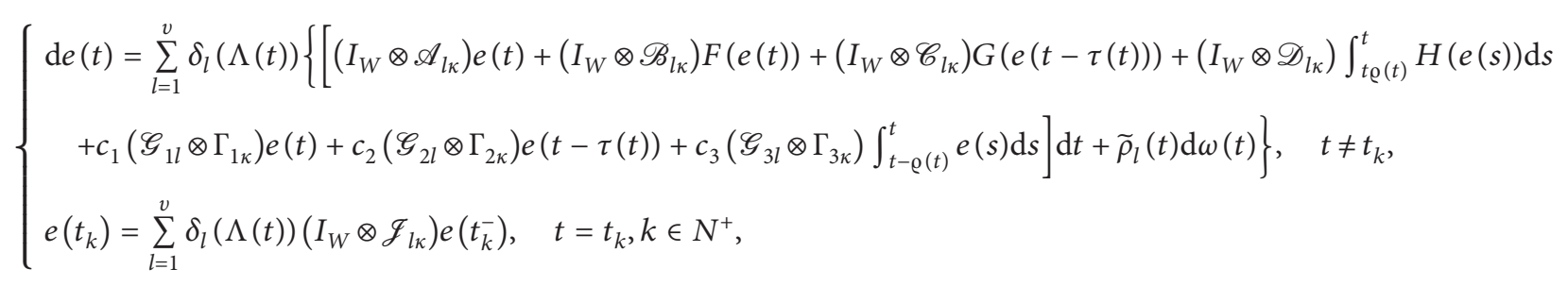

where $I_{W}$ means the $W \times W$ identity matrix.

The following assumptions, definitions, and lemmas are useful in the proof of our main results.
Assumption 1. For all $u, v \in \mathbb{R}^{n}$, the nonlinear functions $f(\cdot), g(\cdot)$ and $h(\cdot)$ are assumed to satisfy the following sector-bounded conditions:

$$
\begin{gathered}
{\left[f(u)-f(v)-\mathscr{X}_{1}(u-v)\right]^{T}\left[f(u)-f(v)-\mathscr{X}_{2}(u-v)\right] \leq 0,} \\
{\left[g(u)-g(v)-\mathscr{Y}_{1}(u-v)\right]^{T}\left[g(u)-g(v)-\mathscr{Y}_{2}(u-v)\right] \leq 0,} \\
{\left[h(u)-h(v)-\mathscr{H}_{1}(u-v)\right]^{T}\left[h(u)-h(v)-\mathscr{H}_{2}(u-v)\right] \leq 0,}
\end{gathered}
$$


where $\mathscr{X}_{1}, \mathscr{Y}_{1}$, and $\mathscr{H}_{1}$ and $\mathscr{X}_{2}, \mathscr{Y}_{2}$, and $\mathscr{H}_{2}$ are real constant matrices with $\mathscr{X}_{2}-\mathscr{X}_{1} \geq 0, \mathscr{Y}_{2}-\mathscr{Y}_{1} \geq 0$ and $\mathscr{H}_{2}-\mathscr{H}_{1} \geq 0$.

Assumption 2. There exist constant matrices $\Upsilon_{l i}(i=1,2,3, l=1,2, \ldots, v)$ with appropriate dimensions so that the following linear growth condition [53]. satisfies

$$
\begin{aligned}
& \quad\left(\rho_{l i}\left(t, p_{1}, q_{1}, r_{1}\right)-\rho_{l j}\left(t, p_{2}, q_{2}, r_{2}\right)\right)^{T} \\
& \quad \times\left(\rho_{l i}\left(t, p_{1}, q_{1}, r_{1}\right)-\rho_{l j}\left(t, p_{2}, q_{2}, r_{2}\right)\right) \\
& \leq\left\|\Upsilon_{l 1}\left(p_{1}-p_{2}\right)\right\|^{2}+\left\|\Upsilon_{l 2}\left(q_{1}-q_{2}\right)\right\|^{2}+\left\|\Upsilon_{l 3}\left(r_{1}-r_{2}\right)\right\|^{2},
\end{aligned}
$$

for all $i, j=1,2, \ldots, N, l=1,2, \ldots, v, \rho_{l i}\left(t, p_{1}, q_{1}, r_{1}\right): \mathbb{R} \times$ $\mathbb{R}^{n} \times \mathbb{R}^{n} \longrightarrow \mathbb{R}^{n}$ and $p_{i}, q_{i}, r_{i}(i=1,2) \in \mathbb{R}^{n}$.

Definition 1 (see [54]). Drive system (9) and response system (12) are said to be stochastically synchronous if error system (17) is stochastically stable, that is, for any initial condition $e(t)=\Phi(t)$ defined on the interval $[-\max \{\tau, \varrho\}, 0]$ and $e(0) \in \mathcal{S}$, the following condition is satisfied:

$$
\lim _{T \rightarrow \infty} \mathbb{E}\left\{\int_{0}^{T} \| e\left((s) \|^{2} \mathrm{~d} s(\Phi(t), \vartheta(0))\right\}<\infty .\right.
$$

Definition 2 (see [55]). The function $\mathscr{V}:\left[t_{0}, \infty\right) \times \mathbb{R}^{n} \times S \longrightarrow \mathbb{R}^{+} \in \Psi_{0}$ whenever

(1) The function $\mathscr{V}$ is continuous on various of the sets $\left[t_{k-1}, t_{k}\right) \times \mathbb{R}^{n} \times S$ and $\forall t \geq t_{0}, \mathscr{V}(t, 0, \kappa) \equiv 0, \kappa \in \mathcal{S}$.

(2) $\mathscr{V}\left(t, e_{1}, \kappa\right)$ is locally Lipschitzian in $e_{1} \in \mathbb{R}^{n}, \kappa \in \mathcal{S}$.

(3) From each $k=1,2, \ldots$, and $\kappa, j \in \mathcal{S}$, there occur finite limits

$$
\begin{aligned}
& \lim _{(t, w, j) \longrightarrow\left(t_{k}^{-}, e_{1}, \kappa\right)} \mathscr{V}\left(t_{k}^{-}, e_{1}, \kappa\right), \\
& \lim _{(t, w, j) \longrightarrow\left(t_{k}^{+}, e_{1}, j\right)} \mathscr{V}\left(t_{k}^{+}, e_{1}, j\right),
\end{aligned}
$$

where $\mathscr{V}\left(t_{k}^{+}, e_{1}, j\right)=\mathscr{V}\left(t_{k}, e_{1}, j\right)$ is convinced.

Definition 3 (see [56]). We introduce stochastic positive functional as $\mathscr{V}[e(t), t, \vartheta(t)=\kappa, t>0]$; the weak infinitesimal operator $\mathscr{L}$ of $\mathscr{V}(e(t), t, \kappa)$ satisfies

$$
\begin{aligned}
& \mathscr{L} \mathscr{V}(e(t), t, \kappa) \triangleq \lim _{\Delta \longrightarrow 0^{+}} \\
& \frac{1}{\Delta} \sup \{\mathbb{E}\{\mathscr{V}(e(t+\Delta), t+\Delta, \kappa) \mid e(t), t, \kappa\}-\mathscr{V}(e(t), t, \kappa)\} .
\end{aligned}
$$

Lemma 1 (see [57]). Let $\otimes$ represent the Kronecker product for which the following properties hold:

$$
\begin{aligned}
& \text { (1) }(\beta \mathscr{X}) \otimes \mathscr{Y}=\mathscr{X} \otimes(\beta \mathscr{Y}) ; \\
& \text { (2) }(\mathscr{X}+\mathscr{Y}) \otimes \mathscr{Z}=\mathscr{X} \otimes \mathscr{Z}+\mathscr{Y} \otimes \mathscr{Z} ; \\
& (3)(\mathscr{X} \otimes \mathscr{Y})(\mathscr{Z} \otimes \mathscr{W})=(\mathscr{X} \mathscr{Z}) \otimes(\mathscr{Y} \mathscr{W}) ; \\
& \text { (4) }(\mathscr{X} \otimes \mathscr{Y})^{T}=\mathscr{X}^{T} \otimes \mathscr{Y}^{T} .
\end{aligned}
$$

Lemma 2 (see [58]). Assume that matrices $\left\{\mathscr{W}_{l}\right\}_{l=1}^{r} \in \mathbb{R}^{n \times m}$ and a positive semidefinite matrix $\mathscr{F} \in \mathbb{R}^{n \times n}$ are given. If $\sum_{l=1}^{r} \delta_{l}(\Lambda(t))=1$ and $0 \leq \delta_{l}(\Lambda(t)) \leq 1$, then

$$
\left(\sum_{l=1}^{r} \delta_{l}(\Lambda(t)) \mathscr{W}_{l}\right)^{T} \mathscr{F}\left(\sum_{l=1}^{r} \delta_{l}(\Lambda(t)) \mathscr{W}_{l}\right) \leq \sum_{l=1}^{r} \delta_{l}(\Lambda(t)) \mathscr{W}_{l}^{T} \mathscr{F}_{\mathscr{W}_{l}} .
$$

Lemma 3 (see [59]). For a positive matrix $\mathcal{O}>0$, scalar $\beta>\beta(t)>0$ and vector function $w:[0, \beta] \longrightarrow \mathbb{R}^{n}$ such that the integrations concerned are well defined; then, the following inequality holds:

$$
\left(\int_{0}^{\beta(t)} w(s) \mathrm{d} s\right)^{T} \mathcal{O}\left(\int_{0}^{\beta(t)} w(s) \mathrm{d} s\right) \leq \beta(t)\left(\int_{0}^{\beta(t)} w^{T}(s) \mathcal{O} w(s)\right) \mathrm{d} s .
$$

Lemma 4 (see [60]). Let $\mathscr{M}=\left(\alpha_{i j}\right)_{W \times W}, \quad \mathcal{S} \in \mathbb{R}^{n \times n}$, $\mathbf{e}=\left(\mathbf{e}_{1}^{T}, \mathbf{e}_{2}^{T}, \ldots, \mathbf{e}_{N}^{T}\right)^{T}$ where $\mathbf{e}_{i}=\left(\mathbf{e}_{i 1}, \mathbf{e}_{i 2}, \ldots, \mathbf{e}_{i n}\right)^{T} \in \mathbb{R}^{n}$ and $\mathbf{z}=\left(\mathbf{z}_{1}^{T}, \mathbf{z}_{2}^{T}, \ldots, \mathbf{z}_{N}^{T}\right)^{T}$ where $\mathbf{z}_{i}=\left(\mathbf{z}_{i 1}, \mathbf{z}_{i 2}, \ldots, \mathbf{z}_{\text {in }}\right)^{T} \in \mathbb{R}^{n}$ with $\mathbf{e}_{k}, \mathbf{z}_{k} \in \mathbb{R}^{n}(k=1,2, \ldots, N)$. If $\mathscr{M}=\mathscr{M}^{T}$ and each row sum of $\mathscr{M}$ is zero, next

$$
\mathbf{e}^{T}(\mathscr{M} \otimes \mathcal{S}) \mathbf{z}=-\sum_{1 \leq i<j \leq N} \alpha_{i j}\left(\mathbf{e}_{i}-\mathbf{e}_{j}\right)^{T} \mathcal{S}\left(\mathbf{z}_{i}-\mathbf{z}_{j}\right) .
$$




\section{Main Results}

Here, some LMI conditions will be created to guarantee that drive framework (9) and response framework (12) are stochastically synchronous by utilizing stochastic Lyapunov-Krasovskii functionals. To introduce the main results of this segment, for simplicity, we denote

$$
\widehat{\mathscr{X}}=\mathscr{X}_{1}^{T} \mathscr{X}_{2}+\mathscr{X}_{2}^{T} \mathscr{X}_{1}, \check{\mathscr{X}}=\mathscr{X}_{1}^{T}+\mathscr{X}_{2}^{T}, \hat{\mathscr{Y}}=\mathscr{Y}_{1}^{T} \mathscr{Y}_{2}+\mathscr{Y}_{2}^{T} \mathscr{Y}_{1}, \check{\mathscr{Y}}=\mathscr{Y}_{1}^{T}+\mathscr{Y}_{2}^{T}, \widehat{\mathscr{X}}=\mathscr{H}_{1}^{T} \mathscr{H}_{2}+\mathscr{H}_{2}^{T} \mathscr{H}_{1}, \check{\mathscr{H}}=\mathscr{H}_{1}^{T}+\mathscr{H}_{2}^{T} .
$$

Theorem 1. Given some positive scalars $\tau>0, \varrho>0$ and $\mu>0$, assume that Assumptions 1 and 2 hold. Then, the T-S fuzzy stochastic complex networks (9) and (12) are stochastically synchronous, if there exist positive definite matrices
$\mathscr{P}_{\kappa}>0(\kappa \in \mathcal{S}), \mathbb{Q}_{1}>0, \mathscr{T}_{1}>0, \mathscr{T}_{2}>0, \mathbb{Q}>0, \mathscr{R}>0, \mathscr{T}>0$, $\mathscr{Z}>0, \mathscr{Z}_{1}>0$, matrices $\mathcal{S}_{i l},(i=1,2,3,4),(l=1,2, \ldots, v)$, and positive scalars $\epsilon, \alpha, \beta, \gamma$ such that the following linear matrix inequalities (LMIs) hold:

$$
\begin{array}{r}
\mathscr{P}_{\kappa}<\epsilon I, \\
\kappa \in \mathcal{S},
\end{array}
$$

$$
\Psi_{l i j}=\left[\begin{array}{cccccccccccccc}
\Psi_{l 11} & \Psi_{l 12} & \Psi_{l 13} & \Psi_{l 14} & \Psi_{l 15} & \Psi_{l 16} & 0 & \Psi_{l 18} & 0 & \Psi_{l 110} & \Psi_{l 111} & \Psi_{l 112} & 0 & 0 \\
* & \Psi_{l 22} & \Psi_{l 23} & 0 & 0 & \Psi_{l 26} & 0 & \Psi_{l 28} & 0 & 0 & \Psi_{l 211} & \Psi_{l 212} & 0 & 0 \\
* & * & \Psi_{l 33} & 0 & 0 & 0 & 0 & 0 & 0 & 0 & 0 & 0 & 0 & 0 \\
* & * & * & \Psi_{l 44} & 0 & 0 & 0 & 0 & 0 & 0 & 0 & 0 & 0 & 0 \\
* & * & * & * & \Psi_{l 55} & 0 & 0 & 0 & 0 & 0 & 0 & 0 & 0 & 0 \\
* & * & * & * & * & \Psi_{l 66} & \Psi_{l 67} & \Psi_{l 68} & \Psi_{l 69} & 0 & 0 & 0 & 0 & 0 \\
* & * & * & * & * & * & * & \Psi_{l 88} & 0 & 0 & 0 & 0 & 0 & 0 \\
* & * & * & * & * & * & * & * & \Psi_{l 99} & 0 & 0 & 0 & 0 & 0 \\
* & * & * & * & * & * & * & * & * & \Psi_{l 1010} & 0 & 0 & 0 & 0 \\
* & * & * & * & * & * & * & * & * & * & \Psi_{l 1111} & 0 & 0 & 0 \\
* & * & * & * & * & * & * & * & * & * & * & \Psi_{l 1212} & 0 & 0 \\
* & * & * & * & * & * & * & * & * & * & * & * & \Psi_{l 1313} & 0 \\
* & * & * & * & * & * & * & * & * & * & * & * & * & \Psi_{l 1414}
\end{array}\right]<,
$$

$\mathscr{J}_{l \kappa}^{T} \mathscr{P}_{j} \mathscr{F}_{l \kappa}-\mathscr{P}_{\kappa} \leq 0, \quad\left[\right.$ here $\left.\vartheta\left(t_{k}\right)=j\right]$, 
where

$$
\begin{aligned}
& \Psi_{l 11}=\mathcal{S}_{3 l} \mathscr{A}_{l \kappa}+\mathscr{A}_{l \kappa}^{T} \mathcal{S}_{3 l}^{T}+c_{1} W \mathscr{G}_{1 l}\left(\mathcal{S}_{3 l} \Gamma_{1 \kappa}+\Gamma_{1 \kappa}^{T} \mathcal{S}_{3 l}\right)+\mathscr{P}_{\kappa} \mathscr{A}_{l \kappa}+\mathscr{A}_{l \kappa}^{T} \mathscr{P}_{\kappa}+c_{1} W \mathscr{G}_{1 l}\left(\mathscr{P}_{\kappa} \Gamma_{1 \kappa}+\Gamma_{1 \kappa}^{T} \mathscr{P}_{\kappa}\right) \\
& +\varrho \mathscr{X}_{1}+\mathcal{S}_{1 l}+\mathcal{S}_{1 l}^{T}-\alpha \widehat{\mathscr{X}}+\mathcal{E} \Upsilon_{1} \Upsilon_{1}^{T}-\beta \widehat{\mathscr{Y}}-\gamma \widehat{\mathscr{X}}+\mathfrak{Q}_{1}+\tau \mathscr{T}_{1}+\sum_{j=1}^{n} \theta_{\kappa j} \mathscr{P}_{j} \\
& \Psi_{l 12}=-\mathcal{S}_{3 l}+\mathscr{A}_{l \kappa}^{T} \mathcal{S}_{4 l}^{T}+\mathcal{S}_{2 l}^{T}+c_{1} W \mathscr{G}_{1 l} \mathcal{S}_{4 l}^{T} \Gamma_{1 \kappa}^{T} \\
& \Psi_{l 13}=\mathcal{S}_{3 l} \mathscr{B}_{l \kappa}+\alpha \check{\mathscr{X}}+\mathscr{P}_{\kappa} \mathscr{B}_{l \kappa} \text {, } \\
& \Psi_{l 14}=\beta \check{Y}, \Psi_{l 15} \\
& \Psi_{l 16}=c_{2} W \mathscr{G}_{2 l}\left(\mathcal{S}_{3 l} \Gamma_{2 \kappa}+\mathscr{P}_{\kappa} \Gamma_{2 \kappa}\right)-\mathcal{S}_{1 l} \text {, } \\
& \Psi_{l 18}=\mathcal{S}_{3 l} \mathscr{C}_{l \kappa}+\mathscr{P}_{\kappa} \mathscr{C}_{l \kappa} \text {, } \\
& \Psi_{l 110}=c_{3} W \mathscr{G}_{3 l}\left(\mathcal{S}_{3 l} \Gamma_{3 \kappa}+\mathscr{P}_{\kappa} \Gamma_{3 \kappa}\right), \\
& \Psi_{l 111}=-\mathcal{S}_{1 l} \text {, } \\
& \Psi_{l 112}=\mathcal{S}_{3 l} \mathscr{D}_{l \kappa}+\mathscr{P}_{\kappa} \mathscr{D}_{l \kappa} \text {, } \\
& \Psi_{l 22}=\tau^{2} \mathscr{Z}-\mathcal{S}_{4 l}-\mathcal{S}_{4 l}^{T} \text {, } \\
& \Psi_{l 23}=\mathcal{S}_{4 l} \mathscr{B}_{l \kappa} \text {, } \\
& \Psi_{l 26}=c_{2} \delta_{4 l} W \mathscr{G}_{2 l} \Gamma_{2 \kappa}-\delta_{2 l} \text {, } \\
& \Psi_{l 28}=\mathcal{S}_{4 l} \mathscr{C}_{l \kappa} \text {, } \\
& \Psi_{l 210}=c_{3} \mathcal{\delta}_{4 l} W \mathscr{G}_{3 l} \Gamma_{3 \kappa} \text {, } \\
& \Psi_{l 211}=-\mathcal{S}_{2 l} \text {, } \\
& \Psi_{l 212}=\mathcal{S}_{4 l} \mathscr{D}_{l \kappa} \text {, } \\
& \Psi_{l 33}=\mathbb{Q}-2 \alpha I, \\
& \Psi_{l 44}=\mathscr{T}-2 \beta I+\tau \mathscr{T}_{2}, \\
& \Psi_{l 55}=\varrho^{2} \mathscr{R}-2 \gamma I, \\
& \Psi_{l 66}=-(1-\mu) Q_{1}-\alpha \widehat{x}-\beta \widehat{\mathscr{Y}}-\gamma \widehat{x}+\varepsilon \Upsilon_{2} \Upsilon_{2}^{T}, \\
& \Psi_{l 67}=\alpha \check{\mathscr{X}}, \\
& \Psi_{l 68}=\beta \check{y}, \\
& \Psi_{l 69}=\gamma \check{\mathscr{H}}, \\
& \Psi_{l 77}=-2 \alpha I-(1-\mu) \mathcal{Q}, \\
& \Psi_{l 88}=-2 \beta I-(1-\mu) \mathcal{T}, \\
& \Psi_{l 99}=-2 \gamma I \text {, } \\
& \Psi_{l 1010}=-\frac{1}{\varrho} \mathscr{Z}_{1}+\varepsilon \Upsilon_{3} \Upsilon_{3}^{T}, \\
& \Psi_{l 1111}=-\mathscr{Z}, \\
& \Psi_{l 1212}=-\mathscr{R}, \\
& \Psi_{l 1313}=-\frac{1}{\tau} \mathscr{T}_{1}, \\
& \Psi_{l 1414}=-\frac{1}{\tau} \mathscr{T}_{2} .
\end{aligned}
$$


Proof. For the sake of notational convenience, we now let

$$
\begin{aligned}
& \mathrm{d} e(t)=\sum_{l=1}^{v} \delta_{l}(\Lambda(t)) z(t) \mathrm{d} t+\sum_{l=1}^{v} \delta_{l}(\Lambda(t)) \widetilde{\rho}_{l}(t) \mathrm{d} \omega(t) \\
& e\left(t_{k}\right)=\sum_{l=1}^{v} \delta_{l}(\Lambda(t))\left(I_{W} \otimes \mathscr{J}_{l \kappa}\right) e\left(t_{k}^{-}\right), \quad k \in N^{+},
\end{aligned}
$$

where

$$
\begin{aligned}
z(t)= & \left(I_{W} \otimes \mathscr{A}_{l \kappa}\right) e(t)+\left(I_{W} \otimes \mathscr{B}_{l \kappa}\right) F(e(t)) \\
& +\left(I_{W} \otimes \mathscr{C}_{l \kappa}\right) G(e(t-\tau(t)))+\left(I_{W} \otimes \mathscr{D}_{l \kappa}\right) \\
& \cdot \int_{t-\varrho(t)}^{t} H(e(s)) \mathrm{d} s+c_{1}\left(\mathscr{G}_{1 l} \otimes \Gamma_{1 \kappa}\right) e(t) \\
& +c_{2}\left(\mathscr{G}_{2 l} \otimes \Gamma_{2 \kappa}\right) e(t-\tau(t))+c_{3}\left(\mathscr{G}_{3 l} \otimes \Gamma_{3 \kappa}\right) \int_{t-\varrho(t)}^{t} e(s) \mathrm{d} s, \\
\tilde{\rho}_{l}(t)= & \rho_{l}\left(t, e(t), e(t-\tau(t)), \int_{t-\varrho(t)}^{t} e(s) \mathrm{d} s\right) .
\end{aligned}
$$

Choose the following Lyapunov-Krasovskii functional where for system

$$
\mathscr{V}(e(t), t, \kappa)=\sum_{o=1}^{9} \mathscr{V}_{o}(e(t), t, \kappa)
$$

$$
\begin{aligned}
& \mathscr{V}_{1}(e(t), t, \kappa)=e^{T}(t)\left(\mathscr{M} \otimes \mathscr{P}_{\kappa}\right) e(t), \\
& \mathscr{V}_{2}(e(t), t, \kappa)=\int_{t-\tau(t)}^{t} e^{T}(s)\left(\mathscr{M} \otimes Q_{1}\right) e(s) \mathrm{d} s, \\
& \mathscr{V}_{3}(e(t), t, \kappa)=\int_{t-\tau(t)}^{t} \int_{\theta}^{t} e^{T}(s)\left(\mathscr{M} \otimes \mathscr{T}_{1}\right) e(s) \mathrm{d} s \mathrm{~d} \theta, \\
& \mathscr{V}_{4}(e(t), t, \kappa)=\int_{t-\tau(t)}^{t} F^{T}(e(s))(\mathscr{M} \otimes \mathscr{Q}) F(e(s)) \mathrm{d} s, \\
& \mathscr{V}_{5}(e(t), t, \kappa)=\int_{t-\tau(t)}^{t} G^{T}(e(s))(\mathscr{M} \otimes \mathscr{T}) G(e(s)) \mathrm{d} s, \\
& \mathscr{V}_{6}(e(t), t, \kappa)=\int_{-\tau(t)}^{0} \int_{t+\theta}^{t} G^{T}(e(s))\left(\mathscr{M} \otimes \mathscr{T}_{2}\right) G(e(s)) \mathrm{d} s \mathrm{~d} \theta, \\
& \mathscr{V}_{7}(e(t), t, \kappa)=\varrho \int_{-\varrho}^{0} \int_{t+\theta}^{t} H^{T}(e(s))(\mathscr{M} \otimes \mathscr{R}) H(e(s)) \mathrm{d} s \mathrm{~d} \theta, \\
& \mathscr{V}_{8}(e(t), t, \kappa)=\tau \int_{-\tau}^{0} \int_{t+\theta}^{t} z^{T}(s)(\mathscr{M} \otimes \mathscr{Z}) z(s) \mathrm{d} s \mathrm{~d} \theta, \\
& \mathscr{V}_{9}(e(t), t, \kappa)=\int_{-\varrho}^{0} \int_{t+\theta}^{t} e^{T}(s)(\mathscr{M} \otimes \mathscr{Z} 1) e(s) \mathrm{d} s \mathrm{~d} \theta .
\end{aligned}
$$


with

$$
\mathscr{M}=\left[\begin{array}{cccc}
W-1 & -1 & \cdots & -1 \\
-1 & W-1 & \cdots & -1 \\
\cdots & \cdots & \cdots & \cdots \\
-1 & -1 & \cdots & W-1
\end{array}\right]_{W \times W}
$$

Observing that $\mathscr{M}_{i l}=\mathscr{G}_{i l} \mathscr{M}=W \mathscr{G}_{i l}(i=1,2,3$, $l=1,2, \ldots$,$) , based on the properties of the Kronecker$ product, for any matrix $\mathscr{H}$ with appropriate dimension, from Lemma 1, we have

$$
(\mathscr{M} \otimes \mathscr{H})\left(\mathscr{G}_{i l} \otimes \Gamma_{i \kappa}\right)=\left(\mathscr{M} \mathscr{G}_{i l}\right) \otimes\left(\mathscr{H} \Gamma_{i \kappa}\right)=\left(W \mathscr{G}_{i l}\right) \otimes\left(\mathscr{H} \Gamma_{i \kappa}\right) .
$$

The stochastic derivative of $\mathscr{V}$ along the trajectories of the network (17) becomes

$$
\begin{aligned}
& \mathscr{L} \mathscr{V}(e(t), t, \kappa)=\sum_{o=1}^{9} \mathscr{L} \mathscr{V}_{o}(e(t), t, \kappa) \\
& \mathscr{L} \mathscr{V}_{1}(e(t), t, \kappa)=2 e^{T}(t)\left(\mathscr{M} \otimes \mathscr{P}_{\kappa}\right) \sum_{l=1}^{v} \delta_{l}(\Lambda(t)) z(t)+\left[\sum_{l=1}^{v} \delta_{l}(\Lambda(t)) \widetilde{\rho}_{l}(t)\right]^{T}\left(\mathscr{M} \otimes \mathscr{P}_{\kappa}\right)\left[\sum_{l=1}^{v} \delta_{l}(\Lambda(t)) \tilde{\rho}_{l}(t)\right] \\
& +e^{T}(t) \sum_{j=1}^{n} \theta_{\kappa j}\left(\mathscr{M} \otimes \mathscr{P}_{j}\right) e(t) \\
& =2 e^{T}(t)\left(\mathscr{M} \otimes \mathscr{P}_{\kappa}\right) \sum_{l=1}^{v} \delta_{l}(\Lambda(t))\left[\left(I_{W} \otimes \mathscr{A}_{l \kappa}\right) e(\Lambda(t))+\left(I_{W} \otimes \mathscr{B}_{l \kappa}\right) F(e(t))+\left(I_{W} \otimes \mathscr{C}_{l \kappa}\right) G(e(t-\tau(t)))\right. \\
& \left.+\left(I_{W} \otimes \mathscr{D}_{l \kappa}\right) \int_{t-\varrho(t)}^{t} H(e(s)) \mathrm{d} s+c_{1}\left(\mathscr{G}_{1 l} \otimes \Gamma_{1 \kappa}\right) e(t)+c_{2}\left(\mathscr{G}_{2 l} \otimes \Gamma_{2 \kappa}\right) e(t-\tau(t))\right] \\
& +c_{3}\left(\mathscr{G}_{3 l} \otimes \Gamma_{3 \kappa}\right) \int_{t-\varrho(t)}^{t} e(s) \mathrm{d} s+\left[\sum_{l=1}^{v} \delta_{l}(\Lambda(t)) \rho_{l}\left(t, e(t), e\left(t-\tau(\Lambda(t)) \rho_{l}\right), \int_{t-\varrho(t)}^{t} e(s) \mathrm{d} s\right)\right]^{T} \\
& \times\left(\mathscr{M} \otimes \mathscr{P}_{\kappa}\right)\left[\sum_{l=1}^{v} \delta_{l}(\Lambda(t)) \rho_{l}\left(t, e(t), e\left(t-\tau(t), \int_{t-\varrho(t)}^{t} e(s) \mathrm{d} s\right)\right)\right] \\
& +e^{T}(t) \sum_{j=1}^{n} \theta_{\kappa j}\left(\mathscr{M} \otimes \mathscr{P}_{j}\right) e(t) \\
& \mathscr{L} \mathscr{V}_{2}(e(t), t, \kappa) \leq e^{T}(t)\left(\mathscr{M} \otimes \mathbb{Q}_{1}\right) e(t)-(1-\mu) e^{T}(t-\tau(t))\left(\mathscr{M} \otimes \mathbb{Q}_{1}\right) e(t-\tau(t)), \\
& \mathscr{L} \mathscr{V}_{3}(e(t), t, \kappa) \leq \tau e^{T}(t)\left(\mathscr{M} \otimes \mathscr{T}_{1}\right) e(t)-\int_{t-\tau(t)}^{t} e^{T}(s)\left(\mathscr{M} \otimes \mathscr{T}_{1}\right) e(s) \mathrm{d} s, \\
& \mathscr{L} \mathscr{V}_{4}(e(t), t, \kappa) \leq F^{T}(e(t))(\mathscr{M} \otimes Q) F(e(t))-(1-\mu) F(e(t-\tau(t)))(\mathscr{M} \otimes \mathscr{Q}) F(e(t-\tau(t))), \\
& \mathscr{L V}_{5}(e(t), t, \kappa) \leq G^{T}(e(t))(\mathscr{M} \otimes \mathscr{T}) G(e(t))-(1-\mu) G^{T}(e(t-\tau(t)))(\mathscr{M} \otimes \mathscr{T}) G(e(t-\tau(t))), \\
& \mathscr{L} \mathscr{V}_{6}(e(t), t, \kappa) \leq \tau G^{T}(e(t))\left(\mathscr{M} \otimes \mathscr{T}_{2}\right) G(e(t))-\int_{t-\tau(t)}^{t} G^{T}(e(s))\left(\mathscr{M} \otimes \mathscr{T}_{2}\right) G(e(s)) \mathrm{d} s, \\
& \mathscr{L} \mathscr{V}_{7}(e(t), t, \kappa)=\varrho^{2} H^{T}(e(t))(\mathscr{M} \otimes \mathscr{R}) H(e(t))-\varrho \int_{t-\varrho}^{t} H^{T}(e(t))(\mathscr{M} \otimes \mathscr{R}) H(e(s)) \mathrm{d} s, \\
& \mathscr{L} \mathscr{V}_{8}(e(t), t, \kappa)=\tau^{2} z^{T}(t)(\mathscr{M} \otimes \mathscr{Z}) z(t)-\tau \int_{t-\tau}^{t} z^{T}(s)(\mathscr{M} \otimes \mathscr{Z}) z(s) \mathrm{d} s, \\
& \mathscr{L} \mathscr{V}_{9}(e(t), t, \kappa)=\varrho e^{T}(t)\left(\mathscr{M} \otimes \mathscr{Z}_{1}\right) e(t)-\int_{t-\varrho}^{t} e^{T}(s)\left(\mathscr{M} \otimes \mathscr{Z}_{1}\right) e(s) \mathrm{d} s .
\end{aligned}
$$


By Lemma 3,

$$
\begin{aligned}
& -\int_{t-\tau(t)}^{t} e^{T}(s)\left(\mathscr{M} \otimes \mathscr{T}_{1}\right) e(s) \mathrm{d} s \leq-\frac{1}{\tau}\left(\int_{t-\tau(t)}^{t} e(s) \mathrm{d} s\right)^{T}\left(\mathscr{M} \otimes \mathscr{T}_{1}\right)\left(\int_{t-\tau(t)}^{t} e(s) \mathrm{d} s\right) \\
& -\int_{t-\tau(t)}^{t} G^{T}(e(s))\left(\mathscr{M} \otimes \mathscr{T}_{2}\right) G(e(s)) \mathrm{d} s \leq-\frac{1}{\tau}\left(\int_{t-\tau(t)}^{t} G(e(s)) \mathrm{d} s\right)^{T}\left(\mathscr{M} \otimes \mathscr{T}_{2}\right)\left(\int_{t-\tau(t)}^{t} G(e(s)) \mathrm{d} s\right), \\
& -\varrho \int_{t-\varrho}^{t} H^{T}(e(s))(\mathscr{M} \otimes \mathscr{R}) H(e(s)) \mathrm{d} s \leq-\left(\int_{t-\varrho(t)}^{t} H(e(s)) \mathrm{d} s\right)^{T}(\mathscr{M} \otimes \mathscr{R})\left(\int_{t-\varrho(t)}^{t} H(e(s)) \mathrm{d} s\right), \\
& -\tau \int_{t-\tau}^{t} z^{T}(s)(\mathscr{M} \otimes \mathscr{Z}) z(s) \mathrm{d} s \leq-\left(\int_{t-\tau(t)}^{t} z(s) \mathrm{d} s\right)^{T}(\mathscr{M} \otimes \mathscr{Z})\left(\int_{t-\tau(t)}^{t} z(s) \mathrm{d} s\right), \\
& -\int_{t-\varrho}^{t} z^{T}(s)\left(\mathscr{M} \otimes \mathscr{Z}_{1}\right) z(s) \mathrm{d} s \quad \leq-\frac{1}{\varrho}\left(\int_{t-\varrho(t)}^{t} z(s) \mathrm{d} s\right)^{T}\left(\mathscr{M} \otimes \mathscr{Z}_{1}\right)\left(\int_{t-\varrho(t)}^{t} z(s) \mathrm{d} s\right) .
\end{aligned}
$$

By Lemma 2, we get

$$
\begin{gathered}
{\left[\sum_{l=1}^{v} \delta_{l}(\Lambda(t)) \rho_{l}\left(t, e(t), e(t-\tau(t)), \int_{t-\varrho(t)}^{t} e(s) \mathrm{d} s\right)\right]^{T}\left(\mathscr{M} \otimes \mathscr{P}_{\kappa}\right)\left[\sum_{l=1}^{\kappa} \delta_{l}(\Lambda(t)) \rho_{l}\left(t, e(t), e(t-\tau(t)), \int_{t-\varrho(t)}^{t} e(s) \mathrm{d} s\right)\right]} \\
\quad \leq \sum_{l=1}^{v} \delta_{l}(\Lambda(t)) \rho_{l}^{T}\left(t, e(t), e(t-\tau(t)), \int_{t-\varrho(t)}^{t} e(s) \mathrm{d} s\right)\left(\mathscr{M} \otimes \mathscr{P}_{\kappa}\right) \rho_{l}\left(t, e(t), e(t-\tau(t)), \int_{t-\varrho(t)}^{t} e(s) \mathrm{d} s\right) .
\end{gathered}
$$

As indicated by the Newton-Leibniz formula, the following equation holds:

$$
2 \sum_{l=1}^{v} \delta_{l}(\Lambda(t))\left[e^{T}(t)\left(\mathscr{M} \otimes \mathcal{S}_{1 l}\right)+z^{T}(t)\left(\mathscr{M} \otimes \mathcal{S}_{2 l}\right)\right]\left[e(t)-e(t-\tau(t))-\int_{t-\tau(t)}^{t} z(s) \mathrm{d} s-\int_{t-\tau(t)}^{t} \tilde{\rho}_{l}(s) \mathrm{d} \omega(s)\right]=0
$$

Also, the following condition holds for any matrices $\mathcal{S}_{3 l}$ and $\mathcal{S}_{4 l}(l=1,2, \ldots, v)$.

$$
\begin{aligned}
& 2 \sum_{l=1}^{v} \delta_{l}(\Lambda(t))\left[e^{T}(t)\left(\mathscr{M} \otimes \mathcal{S}_{3 l}\right)+z^{T}(t)\left(\mathscr{M} \otimes \mathcal{S}_{4 l}\right)\right]\left[\left(I_{W} \otimes \mathscr{A}_{l \kappa}\right) e(t)+\left(I_{W} \otimes \mathscr{B}_{l \kappa}\right) F(e(t))\right. \\
& \quad+\left(I_{W} \otimes \mathscr{C}_{l \kappa}\right) G(e(t-\tau(t)))+\left(I_{W} \otimes \mathscr{D}_{l \kappa}\right) \int_{t-\varrho(t)}^{t} H(e(s)) \mathrm{d} s+c_{1}\left(\mathscr{G}_{1 l} \otimes \Gamma_{1 \kappa}\right) e(t) \\
& \left.\quad+c_{2}\left(\mathscr{G}_{2 l} \otimes \Gamma_{2 \kappa}\right) e(t-\tau(t))+c_{3}\left(\mathscr{G}_{3 l} \otimes \Gamma_{3 \kappa}\right) \int_{t-\varrho(t)}^{t} e(s) \mathrm{d} s-z(t)\right]=0 .
\end{aligned}
$$

Noting that $\mathbb{E}\{\mathrm{d} \omega(t)\}=0$ and combining (39)-(55), using Lemma 1 and Lemma 4, we have 


$$
\begin{aligned}
& \mathscr{L} \mathscr{V}(e(t), t, \kappa) \leq \sum_{l=1}^{v} \delta_{l}(\Lambda(t)) \sum_{i=1}^{N-1} \sum_{j=i+1}^{N}\left[2 \left[\left(e_{i}(t)-e_{j}(\Lambda(t))\right)^{T} \mathscr{P}_{\kappa} \mathscr{A}_{l \kappa}\left(e_{i}(t)-e_{j}(t)\right)+\left(e_{i}(t)-e_{j}(t)\right)^{T} \mathscr{P}_{\kappa} \mathscr{B}_{l \kappa}\right.\right. \\
& \times\left(f\left(e_{i}(t)\right)-f\left(e_{j}(t)\right)\right)+\left(e_{i}(t)-e_{j}(t)\right)^{T} \mathscr{P}_{\kappa} \mathscr{C}_{l \kappa}\left(g\left(e_{i}(t-\tau(t))\right)-g\left(e_{j}(t-\tau(t))\right)\right) \\
& +\left(e_{i}(t)-e_{j}(t)\right)^{T} \mathscr{P}_{\kappa} \mathscr{D}_{l k}\left(\int_{t-\varrho(t)}^{t}\left(h\left(e_{i}(s)\right)-h\left(e_{j}(s)\right)\right) \mathrm{d} s\right)+\left(e_{i}(t)-e_{j}(t)\right)^{T} c_{1} W \mathscr{G}_{1 l} \Gamma_{1 \kappa} \mathscr{P}_{\kappa} \\
& \times\left(e_{i}(t)-e_{j}(t)\right)+\left(e_{i}(t)-e_{j}(t)\right)^{T} c_{2} W \mathscr{G}_{2 l} \Gamma_{2 \kappa} \mathscr{P}_{\kappa}\left(e_{i}(t-\tau(t))-e_{j}(t-\tau(t))\right)+\left(e_{i}(t)-e_{j}(t)\right) \\
& \left.\times c_{3} W \mathscr{G}_{3 l} \Gamma_{3 \kappa} \mathscr{P}_{\kappa}\left(\int_{t-\varrho(t)}^{t}\left(e_{i}(s)-e_{j}(s)\right) \mathrm{d} s\right)\right]+\left(\rho_{l i}\left(t, e_{i}(t), e_{i}(t-\tau(t)), \int_{t-\varrho(t)}^{t} e_{i}(s) \mathrm{d} s\right)\right. \\
& \left.-\rho_{l j}\left(t, e_{j}(t), e_{j}(t-\tau(t)), \int_{t-\varrho(t)}^{t} e_{i}(s) \mathrm{d} s\right)\right)^{T} \mathscr{P}_{k}\left(\rho_{l i}\left(t, e_{i}(t), e_{i}(t-\tau(t)), \int_{t-\varrho(t)}^{t} e_{i}(s) \mathrm{d} s\right)\right. \\
& \left.-\rho_{l j}\left(t, e_{j}(t), e_{j}(t-\tau(t)), \int_{t-\varrho(t)}^{t} e_{i}(s) \mathrm{d} s\right)\right)+\left(e_{i}(t)-e_{j}(t)\right)^{T} Q_{1}\left(e_{i}(t)-e_{j}(t)\right)-(1-\mu) \\
& \times\left(e_{i}(t-\tau(t))-e_{j}(t-\tau(t))\right)^{T} Q_{1}\left(e_{i}(t-\tau(t))-e_{j}(t-\tau(t))\right)+\tau\left(e_{i}(t)-e_{j}(t)\right)^{T} \mathscr{T}_{1}\left(e_{i}(t)-e_{j}(t)\right) \\
& -\frac{1}{\tau}\left(\int_{t-\tau(t)}^{t}\left(e_{i}(s)-e_{j}(s)\right) \mathrm{d} s\right)^{T} \mathscr{T}_{1}\left(\int_{t-\tau(t)}^{t}\left(e_{i}(s)-e_{j}(s)\right) \mathrm{d} s\right)+\left(f\left(e_{i}(t)\right)-f\left(e_{j}(t)\right)\right)^{T} \mathscr{Q} \\
& \times\left(f\left(e_{i}(t)\right)-f\left(e_{j}(t)\right)\right)-(1-\mu)\left(f\left(e_{i}(t-\tau(t))\right)-f\left(e_{j}(t-\tau(t))\right)\right)^{T} Q\left(f\left(e_{i}(t-\tau(t))\right)\right. \\
& \left.-f\left(e_{j}(t-\tau(t))\right)\right)+\left(g\left(e_{i}(t)\right)-g\left(e_{j}(t)\right)\right)^{T} \mathscr{T}\left(g\left(e_{i}(t)\right)-g\left(e_{j}(t)\right)\right)-(1-\mu) \\
& \times\left(g\left(e_{i}(t-\tau(t))\right)-g\left(e_{j}(t-\tau(t))\right)\right)^{T} \mathscr{T}\left(\left(e_{i}(t-\tau(t))\right)-g\left(e_{j}(t-\tau(t))\right)\right) \\
& +\varrho^{2}\left(h\left(e_{i}(t)\right)-h\left(e_{j}(t)\right)\right)^{T} \mathscr{R}\left(h\left(e_{i}(t)\right)-h\left(e_{j}(t)\right)\right)-\left(\int_{t-\varrho(t)}^{t}\left(\left(h\left(e_{i}(s)\right)-h\left(e_{j}(s)\right)\right) \mathrm{d} s\right)^{T}\right. \\
& \times \mathscr{R}\left(\int_{t-\varrho(t)}^{t}\left(\left(h\left(e_{i}(s)\right)-h\left(e_{j}(s)\right)\right) \mathrm{d} s\right)+\tau^{2}\left(z_{i}(t)-z_{j}(t)\right)^{T} \mathscr{Z}\left(z_{i}(t)-z_{j}(t)\right)\right. \\
& -\left(\int_{t-\tau(t)}^{t}\left(z_{i}(s)-z_{j}(s)\right) \mathrm{d} s\right)^{T} \mathscr{Z}\left(\int_{t-\tau(t)}^{t}\left(z_{i}(s)-z_{j}(s)\right) \mathrm{d} s\right)+\left(e_{i}(t)-e_{j}(t)\right)^{T} \mathcal{S}_{1 l} \\
& \times\left(e_{i}(t)-e_{j}(t)-e_{i}(t)-e_{j}(t)\right)^{T} \mathcal{S}_{1 l}\left(e_{i}(t-\tau(t))-e_{j}(t-\tau(t))\right)-\left(e_{i}(t)-e_{j}(t)\right)^{T} \\
& \times \mathcal{S}_{1 l}\left(\int_{t-\tau(t)}^{t}\left(z_{i}(s)-z_{j}(s)\right) \mathrm{d} s\right)+\left(z_{i}(t)-z_{j}(t)\right)^{T} \mathcal{S}_{2 l}\left(e_{i}(t)-e_{j}(t)\right)-\left(z_{i}(t)-z_{j}(t)\right) \mathcal{S}_{2 l} \\
& \times e_{i}(t-\tau(t))-e_{j}(t-\tau(t))-\left(z_{i}(t)-z_{j}(t)\right)^{T} \mathcal{S}_{2 l}\left(\int_{t-\tau(t)}^{t}\left(z_{i}(s)-z_{j}(s)\right) \mathrm{d} s\right) \\
& +\left(e_{i}(t)-e_{j}(t)\right)^{T} \mathcal{S}_{3 l} \mathscr{A}_{l k}\left(e_{i}(t)-e_{j}(t)\right)+\left(e_{i}(t)-e_{j}(t)\right)^{T} \mathcal{S}_{3 l} \mathscr{B}_{l k}\left(f\left(e_{i}(t)\right)-f\left(e_{j}(t)\right)\right)
\end{aligned}
$$




$$
\begin{aligned}
& +\left(e_{i}(t)-e_{j}(t)\right)^{T} \mathcal{S}_{3 l} \mathscr{C}_{l k}\left(g\left(e_{i}(t-\tau(t))\right)-g\left(e_{j}(t-\tau(t))\right)\right)+\left(e_{i}(t)-e_{j}(t)\right)^{T} \mathcal{S}_{3 l} \mathscr{D}_{l \kappa} \\
& \times\left(\int_{t-\varrho(t)}^{t}\left(\left(h\left(e_{i}(s)\right)-h\left(e_{j}(s)\right)\right) \mathrm{d} s\right)+\left(e_{i}(t)-e_{j}(t)\right)^{T} c_{1} W \mathscr{G}_{1 l} \mathcal{S}_{3 l} \Gamma_{1 \kappa}\left(e_{i}(t)-e_{j}(t)\right)\right. \\
& +\left(e_{i}(t)-e_{j}(t)\right)^{T} c_{2} W \mathscr{G}_{2 l} \mathcal{\delta}_{3 l} \Gamma_{2 \kappa}\left(e_{i}(t-\tau(t))-e_{j}(t-\tau(t))\right)+\left(e_{i}(t)\right)-f\left(e_{j}(t)\right)^{T} \\
& \times c_{3} W \mathscr{G}_{3 l} \mathcal{S}_{3 l} \Gamma_{3 \kappa}\left(\int_{t-\varrho(t)}^{t}\left(e_{i}(s)-e_{j}(s) \mathrm{d} s\right)\right)-\left(e_{i}(t)-e_{j}(t)\right)^{T} \mathcal{S}_{3 l}\left(z_{i}(t)-z_{j}(t)\right) \\
& +\left(z_{i}(t)-z_{j}(t)\right)^{T} \mathcal{S}_{4 l} \mathscr{A}_{l k}\left(e_{i}(t)-e_{j}(t)\right)+\left(z_{i}(t)-z_{j}(t)\right)^{T} \mathcal{S}_{4 l} \mathscr{B}_{l k}\left(f\left(e_{i}(t)\right)-f\left(e_{j}(t)\right)\right) \\
& +\left(z_{i}(t)-z_{j}(t)\right)^{T} \mathcal{S}_{4 l} \mathscr{C}_{l k}\left(g\left(e_{i}(t-\tau(t))\right)-g\left(e_{j}(t-\tau(t))\right)\right)+\left(z_{i}(t)-z_{j}(t)\right)^{T} \mathcal{S}_{4 l} \mathscr{D}_{l \kappa} \\
& \times\left(\int_{t-\varrho(t)}^{t}\left(\left(h\left(e_{i}(s)\right)-h\left(e_{j}(s)\right)\right) \mathrm{d} s\right)+\left(z_{i}(t)-z_{j}(t)\right)^{T} c_{1} W \mathscr{G}_{1 l} \Gamma_{1 \kappa} \mathcal{S}_{4 l}\left(e_{i}(t)-e_{j}(t)\right)\right. \\
& +\left(e_{i}(t)-e_{j}(t)\right)^{T} c_{2} W \mathscr{G}_{2 l} \mathcal{S}_{3 l} \Gamma_{2 \kappa}\left(e_{i}(t-\tau(t))\right)-\left(e_{j}(t-\tau(t))\right)+\left(e_{i}(t)-e_{j}(t)\right)^{T} \\
& \times c_{3} W \mathscr{G}_{3 l} \mathcal{S}_{3 l} \Gamma_{3 \kappa}\left(\int_{t-\varrho(t)}^{t} e_{i}(s)-e_{j}(s) \mathrm{d} s\right)-\left(e_{i}(t)-e_{j}(t)\right)^{T} \mathcal{S}_{3 l}\left(z_{i}(t)-z_{j}(t)\right)+\left(z_{i}(t)-z_{j}(t)\right)^{T} \\
& \mathcal{S}_{4 l} \mathscr{A}_{l \kappa}\left(e_{i}(t)-e_{j}(t)\right)+\left(z_{i}(t)-z_{j}(t)\right)^{T} \mathcal{S}_{4 l} \mathscr{B}_{l k}\left(f\left(e_{i}(t)\right)-f\left(e_{j}(t)\right)\right) \\
& +\left(z_{i}(t)-z_{j}(t)\right)^{T} \mathcal{S}_{4 l} \mathscr{C}_{l \kappa}\left(g\left(e_{i}(t-\tau(t))\right)-g\left(e_{j}(t-\tau(t))\right)\right)+\left(z_{i}(t)-z_{j}(t)\right)^{T} \mathcal{S}_{4 l} \mathscr{D}_{l \kappa} \\
& \times\left(\int_{t-\varrho(t)}^{t}\left(\left(h\left(e_{i}(s)\right)-h\left(e_{j}(s)\right)\right) \mathrm{d} s\right)+\left(z_{i}(t)-z_{j}(t)\right)^{T} c_{1} W \mathscr{G}_{1 l} \Gamma_{1 \kappa} \mathcal{S}_{4 l}\left(e_{i}(t)-e_{j}(t)\right)\right. \\
& +\left(z_{i}(t)-z_{j}(t)\right)^{T} \mathcal{S}_{4 l} c_{2} W \mathscr{G}_{2 l} \Gamma_{2 \kappa}\left(e_{i}(t-\tau(t))\right)-\left(e_{j}(t-\tau(t))\right)+\left(z_{i}(t)-z_{j}(t)\right)^{T} \mathcal{S}_{4 l} \mathcal{c}_{3} \\
& \times W \mathscr{G}_{3 l} \Gamma_{3 k}\left(\int_{t-\varrho(t)}^{t} e_{i}(s)-e_{j}(s) \mathrm{d} s\right)-\left(z_{i}(t)-z_{j}(t)\right)^{T} \mathcal{S}_{4 l}\left(z_{i}(t)-z_{j}(t)\right)+\tau\left(g\left(e_{i}(t)\right)-g\left(e_{j}(t)\right)\right)^{T} \\
& \mathscr{T}\left(g\left(e_{i}(t)\right)-g\left(e_{j}(t)\right)\right)-\frac{1}{\tau}\left(\int_{t-\tau(t)}^{t}\left(\left(g\left(e_{i}\right)(s)\right)-g\left(e_{j}(s)\right)\right) \mathrm{d} s\right)^{T} \mathscr{T}_{2} \\
& \times\left(\int_{t-\tau(t)}^{t}\left(\left(g\left(e_{i}(s)\right)-g\left(e_{j}(s)\right)\right) \mathrm{d} s\right)+\varrho\left(e_{i}(t)-e_{j}(t)\right)^{T} \mathscr{Z}_{1}\left(e_{i}(t)-e_{j}(t)\right)\right. \\
& \left.-\frac{1}{\varrho}\left(\int_{t-\varrho(t)}^{t} e_{i}(s)-e_{j}(s) \mathrm{d} s\right)^{T} \mathscr{Z}_{1}\left(\int_{t-\varrho(t)}^{t} e_{i}(s)-e_{j}(s) \mathrm{d} s\right)+\left(e_{i}(t)-e_{j}(t)\right)^{T} \sum_{j=1}^{n} \theta_{\kappa j} \mathscr{P}_{j}\left(e_{i}(t)-e_{j}(t)\right)\right] .
\end{aligned}
$$

Assumption 1 ensures that the following inequality holds:

$$
\alpha\left[\begin{array}{c}
e_{i}(t)-e_{j}(t) \\
f\left(e_{i}(t)\right)-f\left(e_{j}(t)\right)
\end{array}\right]^{T}\left[\begin{array}{cc}
x_{1}^{T} \mathscr{X}_{2}+\mathscr{X}_{2}^{T} \mathscr{X}_{1} & -\left(\mathscr{X}_{1}^{T}+\mathscr{X}_{2}^{T}\right) \\
* & 2 I
\end{array}\right]\left[\begin{array}{c}
e_{i}(t)-e_{j}(t) \\
f\left(e_{i}(t)\right)-f\left(e_{j}(t)\right)
\end{array}\right] \leq 0
$$

i.e., 


$$
\begin{gathered}
\alpha\left[\begin{array}{c}
e_{i}(t)-e_{j}(t) \\
f\left(e_{i}(t)\right)-f\left(e_{j}(t)\right)
\end{array}\right]^{T}\left[\begin{array}{cc}
\widehat{X} & -\check{X} \\
* & 2 I
\end{array}\right]\left[\begin{array}{c}
e_{i}(t)-e_{j}(t) \\
f\left(e_{i}(t)\right)-f\left(e_{j}(t)\right)
\end{array}\right] \leq 0, \quad 1 \leq i<j \leq N, \\
\alpha\left[\begin{array}{c}
e_{i}(t-\tau(t))-e_{j}(t-\tau(t)) \\
f\left(e_{i}(t-\tau(t))\right)-f\left(e_{j}(t-\tau(t))\right)
\end{array}\right]^{T}\left[\begin{array}{cc}
\widehat{\mathscr{X}} & -\check{\mathscr{X}} \\
* & 2 I
\end{array}\right]\left[\begin{array}{c}
e_{i}(t-\tau(t))-e_{j}(t-\tau(t)) \\
f\left(e_{i}(t-\tau(t))\right)-f\left(e_{j}(t-\tau(t))\right)
\end{array}\right] \leq 0,
\end{gathered}
$$

and similarly, one has

$$
\begin{aligned}
& \beta\left[\begin{array}{c}
e_{i}(t)-e_{j}(t) \\
g\left(e_{i}(t)\right)-g\left(e_{j}(t)\right)
\end{array}\right]^{T}\left[\begin{array}{cc}
\hat{y} & \check{Y} \\
* & 2 I
\end{array}\right]\left[\begin{array}{c}
e_{i}(t)-e_{j}(t) \\
g\left(e_{i}(t)\right)-g\left(e_{j}(t)\right)
\end{array}\right] \leq 0, \\
& \beta\left[\begin{array}{c}
e_{i}(t-\tau(t))-e_{j}(t-\tau(t)) \\
g\left(e_{i}(t-\tau(t))\right)-g\left(e_{j}(t-\tau(t))\right)
\end{array}\right]^{T}\left[\begin{array}{cc}
\hat{y} & -\check{y} \\
* & 2 I
\end{array}\right]\left[\begin{array}{c}
e_{i}(t-\tau(t))-e_{j}(t-\tau(t)) \\
g\left(e_{i}(t-\tau(t))\right)-g\left(e_{j}(t-\tau(t))\right)
\end{array}\right] \leq 0, \\
& \gamma\left[\begin{array}{c}
e_{i}(t)-e_{j}(t) \\
h\left(e_{i}(t)\right)-h\left(e_{j}(t)\right)
\end{array}\right]^{T}\left[\begin{array}{cc}
\widehat{x} & -\check{\mathscr{H}} \\
* & 2 I
\end{array}\right]\left[\begin{array}{c}
e_{i}(t)-e_{j}(t) \\
h\left(e_{i}(t)\right)-h\left(e_{j}(t)\right)
\end{array}\right] \leq 0 \\
& \gamma\left[\begin{array}{c}
e_{i}(t-\tau(t))-e_{j}(t-\tau(t)) \\
h\left(e_{i}(t-\tau(t))\right)-h\left(e_{j}(t-\tau(t))\right)
\end{array}\right]^{T}\left[\begin{array}{cc}
\widehat{\mathscr{X}} & -\check{\mathscr{H}} \\
* & 2 I
\end{array}\right]\left[\begin{array}{c}
e_{i}(t-\tau(t))-e_{j}(t-\tau(t)) \\
g\left(e_{i}(t-\tau(t))\right)-g\left(e_{j}(t-\tau(t))\right)
\end{array}\right] \geq 0 .
\end{aligned}
$$

From Assumption 2 and condition (28), we get

$$
\begin{aligned}
& \left(\rho_{l i}\left(t, e_{i}(t), e_{i}(t-\tau(t)), \int_{t-\varrho(t)}^{t} e_{i}(s) \mathrm{d} s\right)-\rho_{l j}\left(t, e_{j}(t), e_{j}(t-\tau(t)), \int_{t-\varrho(t)}^{t} e_{j}(s) \mathrm{d} s\right)\right)^{T} \\
& \quad \times \mathscr{P}_{\kappa}\left(\rho_{l i}\left(t, e_{i}(t), e_{i}(t-\tau(t)), \int_{t-\varrho(t)}^{t} e_{i}(s) \mathrm{d} s\right)-\rho_{l j}\left(t, e_{j}(t), e_{j}(t-\tau(t)), \int_{t-\varrho(t)}^{t} e_{j}(s) \mathrm{d} s\right)\right) \\
& \leq \epsilon\left\{\left(e_{i}(t)-e_{j}(t)\right)^{T} \Upsilon_{1}^{T} \Upsilon_{1}\left(e_{i}(t)-e_{j}(t)\right)+\left(e_{i}(t-\tau(t))-e_{j}(t-\tau(t))\right)^{T} \Upsilon_{2}^{T} \Upsilon_{2}\right. \\
& \left.\quad\left(e_{i}(t-\tau(t))-e_{j}(t-\tau(t))\right)+\left[\int_{t-\varrho(t)}^{t}\left(e_{i}(s)-e_{j}(s)\right) \mathrm{d} s\right]^{T} \Upsilon_{3}^{T} \Upsilon_{3}\left[\int_{t-\varrho(t)}^{t}\left(e_{i}(s)-e_{j}(s)\right) \mathrm{d} s\right]\right\} .
\end{aligned}
$$


Combining equations (56)-(60), with detailed mathematical expectation, we have

$$
\mathbb{E}\{\mathscr{L} \mathscr{V}(e(t), t, \kappa)\} \leq \mathbb{E}\left\{\sum_{l=1}^{v} \delta_{l}(\Lambda(t)) \sum_{i=1}^{N-1} \sum_{j=i+1}^{N} \Omega_{i j}^{T}(t) \Psi_{l i j} \Omega_{i j}(t)\right\}
$$

where

$$
\begin{aligned}
\Omega_{i j}(t)= & {\left[\left(e_{i}(t)-e_{j}(t)\right)^{T}\left(z_{i}(t)-z_{j}(t)\right)^{T}\left(f\left(e_{i}(t)\right)-f\left(e_{j}(t)\right)\right)^{T}\left(g\left(e_{i}(t)\right)-g\left(e_{j}(t)\right)\right)^{T}\left(h\left(e_{i}(t)\right)-h\left(e_{j}(t)\right)\right)^{T}\right.} \\
& \cdot\left(e_{i}(t-\tau(t))-e_{j}(t-\tau(t))\right)^{T}\left(f\left(e_{i}(t-\tau(t))\right)-f\left(e_{j}(t-\tau(t))\right)\right)^{T}\left(g\left(e_{i}(t-\tau(t))\right)-g\left(e_{j}(t-\tau(t))\right)\right)^{T} \\
& \cdot\left(h\left(e_{i}(t-\tau(t))\right)-h\left(e_{j}(t-\tau(t))\right)\right)^{T}\left(\int_{t-\varrho(t)}^{t}\left(e_{i}(s)-e_{j}(s)\right) \mathrm{d} s\right)^{T}\left(\int_{t-\tau(t)}^{t}\left(z_{i}(s)-z_{j}(s)\right) \mathrm{d} s\right)^{T} \\
& \left.\cdot\left(\int_{t-\varrho(t)}^{t}\left(h\left(e_{i}(s)\right)-h\left(e_{j}(s)\right)\right) \mathrm{d} s\right)^{T}\left(\int_{t-\tau(t)}^{t}\left(e_{i}(s)-e_{j}(s)\right) \mathrm{d} s\right)^{T}\left(\int_{t-\tau(t)}^{t}\left(h\left(e_{i}(s)\right)-h\left(e_{j}(s)\right)\right) \mathrm{d} s\right)^{T}\right]^{T} .
\end{aligned}
$$

And $\Psi_{l i j}$ is defined in equation (29). It follows that

$$
\mathbb{E}\{\mathscr{L} \mathscr{V}(e(t), t, \kappa)\} \leq \sum_{l=1}^{v} \delta_{l}(\Lambda(t))\left\{-\eta\|e(t)\|^{2}\right\},
$$

for some $\eta>0$. Now, using Dynkin's formula, we have that for all $T \geq 0$,

$$
\mathbb{E}\{\mathscr{V}(e(T), T, \kappa)\}-\mathbb{E}\{\mathscr{V}(e(0), 0, \kappa)\} \leq-\eta \mathbb{E}\left\{\sum_{l=1}^{v} \delta_{l}(\Lambda(t)) \int_{0}^{T}\|e(s)\|^{2} \mathrm{~d} s\right\}
$$

Hence,

$$
\mathbb{E}\left\{\sum_{l=1}^{v} \delta_{l}(\Lambda(t)) \int_{0}^{T}\|e(s)\|^{2} \mathrm{~d} s\right\} \leq \eta^{-1} \mathbb{E}\{\mathscr{V}(e(0), 0, \kappa)\}
$$

which implies that (20) holds. Therefore, from Definition 1, we have that drive system (9) and response system (12) are stochastically synchronous.

On the other hand, for the impulsive jumping time point $t=t_{k}$, by condition (30), we have 


$$
\begin{aligned}
& \mathscr{V}\left(e\left(t_{k}\right), t_{k}, j\right)-\mathscr{V}\left(e\left(t_{k}^{-}\right), t_{k}^{-}, \kappa\right)=e^{T}\left(t_{k}\right)\left(\mathscr{M} \otimes \mathscr{P}_{j}\right) e\left(t_{k}\right)-e^{T}\left(t_{k}^{-}\right)\left(\mathscr{M} \otimes \mathscr{P}_{k}\right) e\left(t_{k}^{-}\right) \\
& +\int_{t_{k}-\tau\left(t_{k}\right)}^{t_{k}} e^{T}(s)\left(\mathscr{M} \otimes Q_{1}\right) e(s) \mathrm{d} s \\
& +\int_{t_{k}-\tau\left(t_{k}\right)}^{t_{k}} \int_{\theta}^{t_{k}} e^{T}(s)\left(\mathscr{M} \otimes \mathscr{T}_{1}\right) e(s) \mathrm{d} s \mathrm{~d} \theta \\
& +\int_{t_{k}-\tau\left(t_{k}\right)}^{t_{k}} F^{T}(e(s))(\mathscr{M} \otimes Q) F(e(s)) \mathrm{d} s \\
& +\int_{t_{k}-\tau\left(t_{k}\right)}^{t_{k}} G^{T}(e(s))(\mathscr{M} \otimes \mathscr{T}) G(e(s)) \mathrm{d} s \\
& +\int_{-\tau\left(t_{k}\right)}^{0} \int_{t_{k}+\theta}^{t} G^{T}(e(s))\left(M \otimes \mathscr{T}_{2}\right) G(e(s)) \mathrm{d} s \mathrm{~d} \theta \\
& +\varrho \int_{-\varrho}^{0} \int_{t_{k}+\theta}^{t_{k}} H^{T}(e(s))(\mathscr{M} \otimes \mathscr{R}) H(e(s)) \mathrm{d} s \mathrm{~d} \theta \\
& +\tau \int_{-\tau}^{0} \int_{t_{k}+\theta}^{t_{k}} z^{T}(s)(\mathscr{M} \otimes \mathscr{Z}) z(s) \mathrm{d} s \mathrm{~d} \theta \\
& +\int_{-\varrho}^{0} \int_{t_{k}+\theta}^{t_{k}} e^{T}(s)\left(\mathscr{M} \otimes \mathscr{Z}_{1}\right) e(s) \mathrm{d} s \mathrm{~d} \theta \\
& -\int_{t_{k}^{-}-\tau\left(t_{\bar{k}}^{-}\right)}^{t_{\bar{k}}^{-}} e^{T}(s)\left(\mathscr{M} \otimes \mathbb{Q}_{1}\right) e(s) \mathrm{d} s \\
& -\int_{t_{k}^{-}-\tau\left(t_{k}^{-}\right)}^{t_{k}^{-}} \int_{\theta}^{t_{k}^{-}} e^{T}(s)\left(M \otimes \mathscr{T}_{1}\right) e(s) \mathrm{d} s \mathrm{~d} \theta \\
& -\int_{t_{k}^{-}-\tau\left(t_{k}^{-}\right)}^{t_{k}^{-}} F^{T}(e(s))(\mathscr{M} \otimes Q) F(e(s)) \mathrm{d} s \\
& -\int_{t_{k}^{-}-\tau\left(t_{k}^{-}\right)}^{t_{k}^{-}} G^{T}(e(s))(\mathscr{M} \otimes \mathscr{T}) G(e(s)) \mathrm{d} s \\
& -\int_{-\tau\left(t_{k}^{-}\right)}^{t_{t_{k}^{-}}} \int_{t_{k}^{-}+\theta}^{t_{k}^{-}} G^{T}(e(s))\left(M \otimes \mathscr{T}_{2}\right) G(e(s)) \mathrm{d} s \mathrm{~d} \theta \\
& -\varrho \int_{-\varrho}^{0} \int_{t_{k}^{-}+\theta}^{t_{k}^{-}} H^{T}(e(s))(\mathscr{M} \otimes \mathscr{R}) H(e(s)) \mathrm{d} s \mathrm{~d} \theta \\
& -\tau \int_{-\tau}^{0} \int_{t_{k}^{-}+\theta}^{t_{k}^{-}} z^{T}(s)(\mathscr{M} \otimes \mathscr{Z}) z(s) \mathrm{d} s \mathrm{~d} \theta, \\
& -\int_{-\varrho}^{0} \int_{t_{k}^{-}+\theta}^{t_{k}^{-}} e^{T}(s)\left(\mathscr{M} \otimes \mathscr{X}_{1}\right) e(s) \mathrm{d} s \mathrm{~d} \theta \\
& =e^{T}\left(t_{k}\right)\left(\mathscr{M} \otimes \mathscr{P}_{j}\right) e\left(t_{k}\right)-e\left(t_{k}^{-}\right)\left(\mathscr{M} \otimes \mathscr{P}_{k}\right) e\left(t_{k}^{-}\right) .
\end{aligned}
$$

By Lemma 4, we have

$$
\begin{aligned}
\mathscr{V}\left(e\left(t_{k}\right), t_{k}, j\right)-\mathscr{V}\left(e\left(t_{k}^{-}\right), t_{k}^{-}, \kappa\right)= & \left(e_{i}\left(t_{k}^{-}\right)-e_{j}\left(t_{k}^{-}\right)\right)^{T} \mathscr{J}_{l \kappa}^{T} \mathscr{P}_{j} \mathscr{J}_{l k}\left(e_{i}\left(t_{k}^{-}\right)-e_{j}\left(t_{k}^{-}\right)\right) \\
& -\left(e_{i}\left(t_{k}^{-}\right)-e_{j}\left(t_{k}^{-}\right)\right)^{T} \mathscr{P}_{\kappa}\left(e_{i}\left(t_{k}^{-}\right)-e_{j}\left(t_{k}^{-}\right)\right) \\
= & \left(e_{i}\left(t_{k}^{-}\right)-e_{j}\left(t_{k}^{-}\right)\right)^{T}\left[\mathscr{F}_{l \kappa}^{T} \mathscr{P}_{j} \mathscr{J}_{l \kappa}-\mathscr{P}_{\kappa}\right]\left(e_{i}\left(t_{k}^{-}\right)-e_{j}\left(t_{k}^{-}\right)\right) \leq 0,
\end{aligned}
$$

which gives

$$
\mathscr{V}\left(e\left(t_{k}\right), t_{k}, j\right) \leq \mathscr{V}\left(e\left(t_{k}^{-}\right), t_{k}^{-}, \kappa\right)
$$

This completes the proof of Theorem 1.
Remark 1. If there is no effect of the stochastic terms and impulsive control, Markovian jump is not considered, and it can be seen that the aforementioned system (17) is reduced to 


$$
\begin{aligned}
\mathrm{d} e(t)= & \left(I_{W} \otimes \mathscr{A}\right) e(t)+\left(I_{W} \otimes \mathscr{B}\right) F(e(t))+\left(I_{W} \otimes \mathscr{C}\right) G(e(t-\tau(t))) \\
& +\left(I_{W} \otimes \mathscr{D}\right) \int_{t-\varrho(t)}^{t} H(e(s)) \mathrm{d} s+c_{1}\left(\mathscr{G}_{1} \otimes \Gamma_{1}\right) e(t)+c_{2}\left(\mathscr{G}_{2} \otimes \Gamma_{2}\right) e(t-\tau(t))+c_{3}\left(\mathscr{G}_{3} \otimes \Gamma_{3}\right) \int_{t-\varrho(t)}^{t} e(s) \mathrm{d} s
\end{aligned}
$$

Using the same method in Theorem 1, we can get the following results.

Corollary 1. Given some positive scalars $\tau>0, \varrho>0, \mu>0$, assume that Assumption 1 holds. Then, the complex networks (69) is asymptotically stable, if there exist positive definite matrices $\mathscr{P}_{1}>0 \mathbb{Q}_{1}>0, \mathscr{T}_{1}>0, \mathscr{T}_{2}>0, \mathbb{Q}>0, \mathscr{R}>0, \mathscr{T}>0$,
$\mathscr{Z}_{1}>0$, any matrix $\mathcal{S}_{1}$, and positive scalars $\alpha, \beta, \gamma$ such that the following linear matrix inequalities (LMIs) hold:

$$
\Sigma=\left[\Sigma_{l m}\right]_{13 \times 13}<0
$$

where

$$
\begin{aligned}
& \Sigma_{11}=\mathscr{P}_{1} \mathscr{A}+\mathscr{A}^{T} \mathscr{P}_{1}+Q_{1}+\tau \mathscr{T}_{1}+c_{1} W \mathscr{G}_{1}\left(\mathscr{P}_{1} \Gamma_{1}+\Gamma_{1}^{T} \mathscr{P}_{1}\right)+\varrho \mathscr{X}_{1}-\alpha \widehat{\mathscr{X}}-\beta \widehat{\mathscr{Y}}-\gamma \widehat{\mathscr{X}}, \\
& \Sigma_{12}=\mathscr{P}_{1} \mathscr{B}+\alpha \check{\mathscr{X}} \text {, } \\
& \Sigma_{13}=\beta \check{\mathscr{Y}} \text {, } \\
& \Sigma_{14}=\gamma \check{\mathscr{H}} \\
& \Sigma_{15}=c_{2} W \mathscr{G}_{2} \Gamma_{2} \mathscr{P}_{1}+c_{2} W \mathscr{G}_{2} \Gamma_{2}^{T} \mathcal{S}_{1}^{T} \text {, } \\
& \Sigma_{17}=\mathscr{P}_{1} \mathscr{C} \text {, } \\
& \Sigma_{19}=c_{3} W \mathscr{G}_{3 l} \mathscr{P}_{1} \Gamma_{3}+c_{3} W \mathscr{G}_{3} \Gamma_{3}^{T} \delta_{1}^{T} \text {, } \\
& \Sigma_{110}=\mathscr{P}_{1} \mathscr{D} \text {, } \\
& \Sigma_{113}=\mathscr{A}^{T} \mathcal{S}_{1}+W c_{1} \mathscr{G}_{1} \Gamma_{1}^{T} \mathcal{S}_{1}^{T}, \\
& \Sigma_{22}=Q-2 \alpha I \text {, } \\
& \Sigma_{213}=\mathscr{B}^{T} \mathcal{S}_{1} \text {, } \\
& \Sigma_{33}=\mathscr{T}-2 \beta I+\tau \mathscr{T}_{2} \text {, } \\
& \Sigma_{44}=\varrho^{2} \mathscr{R}-2 \gamma I, \\
& \Sigma_{55}=-(1-\mu) Q_{1}-\alpha \widehat{\mathscr{X}}-\beta \hat{\mathscr{Y}}-\gamma \widehat{\mathscr{X}}, \\
& \Sigma_{56}=\alpha \check{\mathscr{X}} \text {, } \\
& \Sigma_{57}=\beta \check{\mathscr{Y}}, \\
& \Sigma_{58}=\beta \check{\mathscr{H}}, \\
& \Sigma_{66}=-2 \alpha I-(1-\mu) \mathbb{Q} \text {, } \\
& \Sigma_{77}=-2 \beta I-(1-\mu) \mathscr{T}, \\
& \Sigma_{713}=\mathscr{C}^{T} \mathcal{S}_{1} \text {, } \\
& \Sigma_{88}=-2 \gamma I \text {, } \\
& \Sigma_{99}=-\frac{1}{\varrho} \mathscr{Z}_{1}, \\
& \Sigma_{1010}=-\mathscr{R}, \\
& \Sigma_{1013}=\mathscr{D}^{T} \mathcal{S}_{1} \text {, } \\
& \Sigma_{1111}=-\frac{1}{\tau} \mathscr{T}_{1}, \\
& \Sigma_{1212}=-\frac{1}{\tau} \mathscr{T}_{2}, \\
& \Sigma_{1313}=-\mathcal{S}_{1}-\mathcal{S}_{1}^{T} \text {. }
\end{aligned}
$$


Proof. Consider the equivalent LKF in Theorem 1. Eliminating the stochastic term and following the equivalent argument of Theorem 1, we can see that the following condition is satisfied for any matrix $\mathcal{S}_{1}$ with suitable measurement:

$$
\begin{aligned}
& 2 \dot{e}^{T}(t)\left(I_{W} \otimes \mathcal{S}_{1}\right)\left[-\dot{e}(t)-\left(I_{W} \otimes \mathscr{A}\right) e(t)+\left(I_{W} \otimes \mathscr{B}\right) F(e(t))+\left(I_{W} \otimes \mathscr{C}\right) G(e(t-\tau(t)))\right. \\
& \quad+\left(I_{W} \otimes \mathscr{D}\right) \int_{t-\varrho(t)}^{t} H(e(s)) \mathrm{d} s+c_{1}\left(\mathscr{G}_{1} \otimes \Gamma_{1}\right) e(t)+c_{2}\left(\mathscr{G}_{2} \otimes \Gamma_{2}\right) e(t-\tau(t)) \\
& \left.\quad+c_{3}\left(\mathscr{G}_{3} \otimes \Gamma_{3}\right) \int_{t-\varrho(t)}^{t} e(s) \mathrm{d} s\right]=0,
\end{aligned}
$$

which implies that

$$
\mathbb{E}\{\mathscr{L} \mathscr{V}(e(t), t)\} \leq \mathbb{E}\left\{\Omega_{i j}(t)^{T} \Sigma \Omega_{i j}(t)\right\},
$$

where

$$
\begin{aligned}
\Omega_{i j}(t)= & {\left[\left(e_{i}(t)-e_{j}(t)\right)^{T}\left(f\left(e_{i}(t)\right)-f\left(e_{j}(t)\right)\right)^{T}\left(g\left(e_{i}(t)\right)-g\left(e_{j}(t)\right)\right)^{T}\left(h\left(e_{i}(t)\right)-h\left(e_{j}(t)\right)\right)^{T}\right.} \\
& \cdot\left(e_{i}(t-\tau(t))-e_{j}(t-\tau(t))\right)^{T}\left(f\left(e_{i}(t-\tau(t))\right)-f\left(e_{j}(t-\tau(t))\right)\right)^{T}\left(g\left(e_{i}(t-\tau(t))\right)-g\left(e_{j}(t-\tau(t))\right)\right)^{T} \\
& \cdot\left(h\left(e_{i}(t-\tau(t))\right)-h\left(e_{j}(t-\tau(t))\right)\right)^{T}\left(\int_{t-\varrho(t)}^{t}\left(e_{i}(s)-e_{j}(s)\right) \mathrm{d} s\right)^{T}\left(\int_{t-\varrho(t)}^{t}\left(h\left(e_{i}(s)\right)-h\left(e_{j}(s)\right)\right) \mathrm{d} s\right)^{T} \\
& \left.\cdot\left(\int_{t-\tau(t)}^{t}\left(e_{i}(s)-e_{j}(s)\right) \mathrm{d} s\right)^{T}\left(\int_{t-\tau(t)}^{t}\left(g\left(e_{i}(s)\right)-g\left(e_{j}(s)\right)\right) \mathrm{d} s\right)^{T}\left(\dot{e}_{i}(t)-\dot{e}_{j}(t)\right)^{T}\right]^{T} .
\end{aligned}
$$

At that point, similar to the proof of Theorem 1, we can get that framework (69) is asymptotically stable. This complete the proof.

Remark 2. In the past few years, the fuzzy logic theory has been seen to be effective in dealing with different types of complex nonlinear systems, which has received a great deal of attention in the literature [3, 61-64]. The T-S fuzzy model is one of the most popular models in the fuzzy systems. In this type of fuzzy models, a nonlinear system is represented by a set of local linear models smoothly connected by nonlinear membership functions, which has a convenient and simple dynamic structure, so the existing results for linear systems theory can be readily extended for the class of nonlinear systems. In this article, a general T-S fuzzy stochastic complex network with mixed time-varying delays is introduced.
Remark 3. In practice, due to the limitations of equipment and the disturbances of environment, the perfect information is rarely available. Thus, it is practical meaning to consider more constraints for fuzzy complex networks. However, in $[65,66]$, the sampled-data synchronization problem of complex networks with T-S fuzzy nodes has been investigated, but the mixed time-varying delays are not considered, which may be restrictive in many practical applications. Hence, it is very interesting to consider mixed time-varying coupling delays and not consider sampled data in fuzzy complex networks.

Remark 4. In order to show the reduced conservatism of our stability criteria, we consider the following system as a special case of system (17) which reduces to a delayed complex network described by

$$
\begin{aligned}
\operatorname{de}(t)= & \sum_{l=1}^{v} \delta_{l}(\Lambda(t))\left\{\left[\left(I_{W} \otimes \mathscr{A}_{l}\right) e(t)+\left(I_{W} \otimes \mathscr{B}_{l}\right) F(e(t))+\left(I_{W} \otimes \mathscr{C}_{l}\right) G(e(t-\tau(t)))+\left(I_{W} \otimes \mathscr{D}_{l}\right) \int_{t-\varrho(t)}^{t} H(e(s)) \mathrm{d} s\right.\right. \\
& \left.\left.+\left(\mathscr{G}_{1 l} \otimes \Gamma_{1}\right) e(t)+\left(\mathscr{G}_{2 l} \otimes \Gamma_{2}\right) e(t-\tau(t))\right] \mathrm{d} t+\widetilde{\rho}_{l}(t) \mathrm{d} \omega(t)\right\} .
\end{aligned}
$$


The proof follows similar ideas in to Theorem 1 and Corollary 1. Now, we present the result in the following corollary.

Corollary 2. Given some positive scalars $\tau>0, \varrho>0$, and $\mu>0$, assume that Assumptions 1 and 2 hold. Then, the T-S fuzzy stochastic complex networks (9) and (12) are stochastically synchronous, if there exist positive definite matrices $\mathscr{P}_{1}>0, \mathscr{Q}_{1}>0, \mathscr{T}_{1}>0, \mathscr{T}_{2}>0, \mathscr{Q}>0, \mathscr{R}>0, \mathscr{T}>0, \mathscr{Z}>0$, $\mathscr{Z}_{1}>0$, matrices $\mathcal{S}_{i l}, \quad(i=1,2,3,4), \quad(l=1,2, \ldots, v)$, and positive scalars $\epsilon, \alpha, \beta, \gamma$ such that the following linear matrix inequalities (LMIs) hold:

$$
\begin{gathered}
<\mathscr{P}_{1} \in I, \\
\kappa \in \mathcal{S}, \\
\widetilde{\Psi}=[\widetilde{\Psi}]_{14 \times 14}<0,
\end{gathered}
$$

where

$$
\begin{aligned}
& \widetilde{\Psi}_{l 11}=\mathcal{S}_{3 l} \mathscr{A}_{l}+\mathscr{A}_{l}^{T} \mathcal{\delta}_{3 l}^{T}+W \mathscr{G}_{1 l}\left(\mathcal{S}_{3 l} \Gamma_{1}+\Gamma_{1}^{T} \mathcal{S}_{3 l}\right)+\mathscr{P}_{1} \mathscr{A}_{l}+\mathscr{A}_{l}^{T} \mathscr{P}_{1}+W \mathscr{G}_{1 l}\left(\mathscr{P}_{1} \Gamma_{1}+\Gamma_{1}^{T} \mathscr{P}_{1}\right) \\
& +\varrho \mathscr{X}_{1}+\mathcal{S}_{1 l}+\mathcal{S}_{1 l}^{T}-\alpha \hat{\mathscr{X}}+\epsilon \Upsilon_{1} \Upsilon_{1}^{T}-\beta \hat{\mathscr{Y}}-\gamma \widehat{\mathscr{X}}+Q_{1}+\tau \mathscr{T}_{1}, \widetilde{\Psi}_{l 12}=-\mathcal{S}_{3 l}+\mathscr{A}_{l}^{T} \mathcal{S}_{4 l}^{T}+\mathcal{S}_{2 l}^{T}+W \mathscr{G}_{1 l} \mathcal{S}_{4 l}^{T} T_{1}^{T}, \\
& \widetilde{\Psi}_{l 13}=\mathcal{S}_{3 l} \mathscr{B}_{l}+\alpha \check{\mathscr{X}}+\mathscr{P}_{1} \mathscr{B}_{l}, \widetilde{\Psi}_{l 14}=\beta \check{\mathscr{Y}}, \widetilde{\Psi}_{l 15}=\gamma \check{\mathscr{H}}, \widetilde{\Psi}_{l 16}=W \mathscr{G}_{2 l}\left(\mathcal{S}_{3 l} \Gamma_{2}+\mathscr{P}_{1} \Gamma_{2}\right)-\mathcal{S}_{1 l}, \widetilde{\Psi}_{l 18}=\mathcal{S}_{3 l} \mathscr{C}_{l}+\mathscr{P}_{1} \mathscr{C}_{l}, \\
& \widetilde{\Psi}_{l 111}=-\mathcal{S}_{1 l}, \widetilde{\Psi}_{l 112}=\mathcal{S}_{3 l} \mathscr{D}_{l}+\mathscr{P}_{1} \mathscr{D}_{l}, \widetilde{\Psi}_{l 22}=\tau^{2} \mathscr{Z}-\mathcal{S}_{4 l}-\mathcal{S}_{4 l}^{T}, \widetilde{\Psi}_{l 23}=\mathcal{S}_{4 l} \mathscr{B}_{l}, \widetilde{\Psi}_{l 26}=\mathcal{S}_{4 l} W \mathscr{G}_{2 l} \Gamma_{2}-\mathcal{S}_{2 l} \text {, } \\
& \widetilde{\Psi}_{l 28}=\mathcal{S}_{4 l} \mathscr{C}_{l}, \widetilde{\Psi}_{l 211}=-\mathcal{S}_{2 l}, \widetilde{\Psi}_{l 212}=\mathcal{S}_{4 l} \mathscr{D}_{l}, \widetilde{\Psi}_{l 33}=\mathscr{Q}-2 \alpha I, \widetilde{\Psi}_{l 44}=\mathscr{T}-2 \beta I+\tau \mathscr{T}_{2}, \widetilde{\Psi}_{l 55}=\varrho^{2} \mathscr{R}-2 \gamma I, \\
& \widetilde{\Psi}_{l 66}=-(1-\mu) Q_{1}-\alpha \widehat{\mathscr{X}}-\beta \hat{\mathscr{Y}}-\gamma \widehat{\mathscr{X}}+\epsilon \Upsilon_{2} \Upsilon_{2}^{T}, \widetilde{\Psi}_{l 67}=\alpha \check{\mathscr{X}}, \widetilde{\Psi}_{l 68}=\beta \check{\mathscr{Y}}, \widetilde{\Psi}_{l 69}=\gamma \check{\mathscr{H}}, \widetilde{\Psi}_{l 77}=-2 \alpha I-(1-\mu) \mathscr{Q}, \\
& \widetilde{\Psi}_{l 88}=-2 \beta I-(1-\mu) \mathscr{T}, \widetilde{\Psi}_{l 99}=-2 \gamma I, \widetilde{\Psi}_{l 1010}=-\frac{1}{\varrho} \mathscr{Z}{ }_{1}+\epsilon \Upsilon_{3} \Upsilon_{3}^{T}, \widetilde{\Psi}_{l 1111}=-\mathscr{Z}, \widetilde{\Psi}_{l 1212}=-\mathscr{R}, \widetilde{\Psi}_{l 1313}=-\frac{1}{\tau} \mathscr{T}_{1}, \widetilde{\Psi}_{l 1414}=-\frac{1}{\tau} \mathscr{T}_{2} .
\end{aligned}
$$

\section{Numerical Examples}

In this section, numerical models are displayed to show the adequacy of the results determined previously.

Example 1. We consider the following stochastic T-S fuzzy complex systems with mixed time-varying delays and
Markovian jumping parameters with 3 nodes and $\kappa=\{1,2\}$, $l=\{1,2\}$.

Fuzzy rule 1:

IF $\Lambda_{1}(t)=x_{11}(t)$ is $\chi_{1}^{1}\left(x_{11}(t)\right)$

$$
\begin{aligned}
\mathrm{d} x_{i}(t)= & {\left[\mathscr{A}_{11} x_{i}(t)+\mathscr{B}_{11} f\left(x_{i}(t)\right)+\mathscr{C}_{11} g\left(x_{i}(t-\tau(t))\right)+\mathscr{D}_{11} \int_{t-\varrho(t)}^{t} h\left(x_{i}(s)\right) \mathrm{d} s+c_{1} \mathscr{G}_{11 i j} \Gamma_{11} x_{i}(t)\right.} \\
& \left.+c_{2} \mathscr{G}_{21 i j} \Gamma_{21} x_{i}(t-\tau(t))+c_{3} \mathscr{G}_{31 i j} \Gamma_{31} \int_{t-\varrho(t)}^{t} x_{i}(s) \mathrm{d} s\right] \mathrm{d} t \\
& +\rho_{1 i}\left(t, x_{i}(t), x_{i}\left(t-\tau(t), \int_{t-\varrho(t)}^{t} x_{i}(s) \mathrm{d} s\right) \mathrm{d} \omega(t), \quad t \neq t_{k}, t \geq t_{0},\right. \\
x_{i}\left(t_{k}\right)= & \mathscr{F}_{11} x_{i}\left(t_{k}^{-}\right), \quad i=1,2, \ldots, N, t=t_{k},
\end{aligned}
$$


Fuzzy rule 2:

THEN

$$
\text { IF } \Lambda_{1}(t)=x_{11}(t) \text { is } \chi_{1}^{2}\left(x_{11}(t)\right)
$$

$$
\begin{aligned}
\mathrm{d} x_{i}(t)= & {\left[\mathscr{A}_{21} x_{i}(t)+\mathscr{B}_{21} f\left(x_{i}(t)\right)+\mathscr{C}_{21} g\left(x_{i}(t-\tau(t))\right)+\mathscr{D}_{21} \int_{t-\varrho(t)}^{t} h\left(x_{i}(s)\right) \mathrm{d} s+c_{1} \mathscr{G}_{11 i j} \Gamma_{11} x_{i}(t)\right.} \\
& \left.+c_{2} \mathscr{G}_{22 i j} \Gamma_{21} x_{i}(t-\tau(t))+c_{3} \mathscr{G}_{32 i j} \Gamma_{31} \int_{t-\varrho(t)}^{t} x_{i}(s) \mathrm{d} s\right] \mathrm{d} t \\
& +\rho_{2 i}\left(t, x_{i}(t) x_{i}\left(t-\tau(t), \int_{t-\varrho(t)}^{t} x_{i}(s) \mathrm{d} s\right) \mathrm{d} \omega(t), \quad t \neq t_{k}, t \geq t_{0}\right. \\
x_{i}\left(t_{k}\right)= & \mathscr{J}_{21} x_{i}\left(t_{k}^{-}\right), \quad i=1,2, \ldots, N, t=t_{k} .
\end{aligned}
$$

Fuzzy Rule 1:

THEN

IF $\Lambda_{1}(t)=x_{11}(t)$ is $\chi_{1}^{1}\left(x_{11}(t)\right)$

$$
\begin{aligned}
\mathrm{d} x_{i}(t)= & {\left[\mathscr{A}_{12} x_{i}(t)+\mathscr{B}_{12} f\left(x_{i}(t)\right)+\mathscr{C}_{12} g\left(x_{i}(t-\tau(t))\right)\right.} \\
& \left.+\mathscr{D}_{12} \int_{t-\varrho(t)}^{t} h\left(x_{i}(s)\right) \mathrm{d} s+c_{1} \mathscr{G}_{12 i j} \Gamma_{12} x_{i}(t)+c_{2} \mathscr{G}_{21 i j} \Gamma_{22} x_{i}(t-\tau(t))+c_{3} \mathscr{G}_{31 i j} \Gamma_{32} \int_{t-\varrho(t)}^{t} x_{i}(s) \mathrm{d} s\right] \mathrm{d} t \\
& +\rho_{1 i}\left(t, x_{i}(t), x_{i}\left(t-\tau(t), \int_{t-\varrho(t)}^{t} x_{i}(s) \mathrm{d} s\right) \mathrm{d} \omega(t), \quad t \neq t_{k}, t \geq t_{0} \cdot x_{i}\left(t_{k}\right)=\mathscr{J}_{12} x_{i}\left(t_{k}^{-}\right), \quad i=1,2, \ldots, N, t=t_{k},\right.
\end{aligned}
$$

Fuzzy rule 2:

\section{THEN}

$$
\text { IF } \Lambda_{1}(t)=x_{11}(t) \text { is } \chi_{1}^{2}\left(x_{11}(t)\right)
$$

$$
\begin{aligned}
\mathrm{d} x_{i}(t)= & {\left[\mathscr{A}_{22} x_{i}(t)+\mathscr{B}_{22} f(x(t))+\mathscr{C}_{22} g\left(x_{i}(t-\tau(t))\right)+\mathscr{D}_{22} \int_{t->(t)}^{t} h\left(x_{i}(s)\right) \mathrm{d} s+c_{1} \mathscr{G}_{12 i j} \Gamma_{12} x_{i}(t)\right.} \\
& \left.+c_{2} \mathscr{G}_{22 i j} \Gamma_{22} x_{i}(t-\tau(t))+c_{3} \mathscr{G}_{32 i j} \Gamma_{32} \int_{t-\varrho(t)}^{t} x_{i}(s) \mathrm{d} s\right] \mathrm{d} t \\
& +\rho_{2 i}\left(t, x_{i}(t), x_{i}\left(t-\tau(t), \int_{t-\varrho(t)}^{t} x_{i}(s) \mathrm{d} s\right) \mathrm{d} \omega(t), \quad t \neq t_{k}, t \geq t_{0},\right. \\
x_{i}\left(t_{k}\right)= & \mathscr{J}_{22} x_{i}\left(t_{k}^{-}\right), \quad i=1,2, \ldots, N, t=t_{k},
\end{aligned}
$$


where $x(t)=\left(x_{i 1}(t), x_{i 2}(t)\right)^{T}(i=1,2)$ and effective relevant parameters are given as follows.

Mode 1:

$$
\begin{aligned}
& \mathscr{A}_{11}=\left[\begin{array}{cc}
1.5 & 0 \\
0 & 1.5
\end{array}\right], \\
& \mathscr{B}_{11}=\left[\begin{array}{cc}
-0.01 & -0.08 \\
0.06 & 0.019
\end{array}\right] \text {, } \\
& \mathscr{C}_{11}=\left[\begin{array}{ll}
0.03 & 0.02 \\
0.06 & 0.021
\end{array}\right] \text {, } \\
& \mathscr{D}_{11}=\left[\begin{array}{cc}
-0.025 & -0.01 \\
0.3 & 0.075
\end{array}\right] \text {, } \\
& \mathscr{A}_{21}=\left[\begin{array}{cc}
-1 & 0.2 \\
0 & -1
\end{array}\right] \text {, } \\
& \mathscr{B}_{21}=\left[\begin{array}{cc}
1.8 & -0.12 \\
-5.2 & 3.5
\end{array}\right] \text {, } \\
& \mathscr{C}_{21}=\left[\begin{array}{ll}
0.93 & 0.62 \\
1.06 & 0.021
\end{array}\right] \text {, } \\
& \mathscr{D}_{21}=\left[\begin{array}{cc}
-3.025 & -1.01 \\
-0.3 & 3.075
\end{array}\right] \text {, } \\
& \Gamma_{11}=\left[\begin{array}{cc}
0.8 & 0 \\
0 & 0.8
\end{array}\right] \text {, } \\
& \Gamma_{21}=\left[\begin{array}{ll}
1 & 0 \\
0 & 1
\end{array}\right] \text {, } \\
& \Gamma_{31}=\left[\begin{array}{cc}
0.5 & 0 \\
0 & 0.5
\end{array}\right] \text {, } \\
& \mathscr{F}_{11}=\left[\begin{array}{cc}
0.4 & 0 \\
0 & 0.4
\end{array}\right] \text {, } \\
& \mathscr{F}_{21}=\left[\begin{array}{cc}
0.4 & 0 \\
0 & 0.4
\end{array}\right] \text {, } \\
& \mathscr{G}_{11}=\left[\begin{array}{ccc}
-0.2 & 0.1 & 0.1 \\
0.1 & -0.2 & 0.1 \\
0.1 & 0.1 & -0.2
\end{array}\right] \text {, } \\
& \mathscr{G}_{21}=\left[\begin{array}{ccc}
-0.3 & 0.15 & 0.15 \\
0.15 & -0.3 & 0.15 \\
0.15 & 0.15 & -0.3
\end{array}\right] \text {, } \\
& \mathscr{G}_{31}=\left[\begin{array}{ccc}
-1 & 0 & 1 \\
0 & -1 & 1 \\
1 & 1 & -2
\end{array}\right] \text {. }
\end{aligned}
$$

Mode 2:

$$
\begin{aligned}
& \mathscr{A}_{12}=\left[\begin{array}{ll}
2 & 0 \\
0 & 2
\end{array}\right], \\
& \mathscr{B}_{12}=\left[\begin{array}{ll}
0.15 & -1.08 \\
0.75 & 2.019
\end{array}\right] \text {, } \\
& \mathscr{C}_{12}=\left[\begin{array}{ll}
0.23 & 1.02 \\
0.56 & 1.021
\end{array}\right] \text {, } \\
& \mathscr{D}_{12}=\left[\begin{array}{cc}
-0.025 & -1.01 \\
-1.3 & 0.75
\end{array}\right] \text {, } \\
& \mathscr{A}_{22}=\left[\begin{array}{cc}
1.65 & 0.2 \\
0 & 1.65
\end{array}\right] \text {, } \\
& \mathscr{B}_{22}=\left[\begin{array}{cc}
1.15 & -1.08 \\
0.85 & 1.019
\end{array}\right] \text {, } \\
& \mathscr{C}_{22}=\left[\begin{array}{cc}
-1.23 & 0.02 \\
1.56 & 0.021
\end{array}\right] \text {, } \\
& \mathscr{D}_{22}=\left[\begin{array}{cc}
-1.025 & -0.01 \\
-2.3 & 1.75
\end{array}\right] \text {, } \\
& \Gamma_{12}=\left[\begin{array}{cc}
0.5 & 0 \\
0 & 0.5
\end{array}\right] \text {, } \\
& \Gamma_{22}=\Gamma_{32}=\left[\begin{array}{ll}
1 & 0 \\
0 & 1
\end{array}\right], \\
& \mathscr{F}_{12}=\left[\begin{array}{cc}
0.4 & 0 \\
0 & 0.4
\end{array}\right] \text {, } \\
& \mathscr{J}_{22}=\left[\begin{array}{cc}
0.4 & 0 \\
0 & 0.4
\end{array}\right] \text {, } \\
& \mathscr{G}_{12}=\left[\begin{array}{ccc}
-2 & 2 & 0 \\
0 & -2 & 2 \\
2 & 0 & -2
\end{array}\right] \text {, } \\
& \mathscr{G}_{22}=\left[\begin{array}{ccc}
-2 & 1 & 0 \\
0 & -2 & 1 \\
1 & 0 & -2
\end{array}\right] \text {, } \\
& \mathscr{G}_{32}=\left[\begin{array}{ccc}
-1 & 1 & 0 \\
3 & -1 & -2 \\
0 & 1 & -1
\end{array}\right] \text {. }
\end{aligned}
$$


The transition rate matrix with two operation modes is

$$
\Theta=\left[\begin{array}{cc}
-3 & 6 \\
4 & -7
\end{array}\right] \text {. }
$$

Let $\varrho=1, \tau=1.5, \mu=0.5, W=3, c_{1}=c_{2}=c_{3}=1$. The nonlinear vector-valued functions are

$$
\begin{aligned}
f\left(x_{i}(t)\right) & =\left(\tanh \left(0.2 x_{i 1}(t)\right),-\tanh \left(0.75 x_{i 2}(t)\right)\right)^{T}, \\
g\left(x_{i}(t)\right) & =h\left(x_{i}(t)\right) \\
& =\left(0.2 x_{i 1}(k)-\tanh \left(0.1 x_{i 1}(t)\right), 0.1 x_{i 2}(t)\right)^{T} .
\end{aligned}
$$

The other parameters are chosen as follows:

$$
\begin{aligned}
\mathscr{X}_{1} & =\left[\begin{array}{cc}
0.3 & 0.2 \\
0 & 0.2
\end{array}\right], \\
\mathscr{Y}_{1} & =\left[\begin{array}{cc}
0.1 & 0 \\
0 & 0.1
\end{array}\right], \\
\mathscr{H}_{1} & =\left[\begin{array}{cc}
0.1 & 0 \\
0 & 0.1
\end{array}\right], \\
\mathscr{X}_{2} & =\left[\begin{array}{cc}
0.5 & 0.2 \\
0 & 0.95
\end{array}\right], \\
\mathscr{Y}_{2} & =\left[\begin{array}{cc}
0.2 & 0 \\
0 & 0.1
\end{array}\right], \\
\mathscr{H}_{2} & =\left[\begin{array}{cc}
0.2 & 0 \\
0 & 0.1
\end{array}\right], \\
\Sigma_{1} & =\Sigma_{2}=\Sigma_{3}=\left[\begin{array}{cc}
0.03 & 0 \\
0 & 0.03
\end{array}\right] .
\end{aligned}
$$

Solving LMIs (28)-(30) in Theorem 1, we can obtain the feasible solutions as follows:

$$
\begin{aligned}
& \mathscr{P}_{1}=10^{-4}\left[\begin{array}{cc}
0.3928 & -0.4520 \\
-0.4520 & 0.7102
\end{array}\right], \\
& \mathscr{P}_{2}=10^{-4}\left[\begin{array}{cc}
0.3381 & -0.4489 \\
-0.4489 & 0.7351
\end{array}\right], \\
& \mathscr{Q}_{1}=10^{-3}\left[\begin{array}{cc}
-0.1152 & -0.1070 \\
-0.1070 & 0.1014
\end{array}\right], \\
& \mathscr{T}_{1}=10^{-4}\left[\begin{array}{cc}
0.1915 & -0.1051 \\
-0.1051 & 0.1581
\end{array}\right], \\
& \mathscr{T}_{2}=\left[\begin{array}{cc}
0.0013 & -0.0001 \\
-0.0001 & 0.0014
\end{array}\right], \\
& Q_{2}=10^{-3}\left[\begin{array}{cc}
-0.0933 & 0.0844 \\
0.0844 & -0.1099
\end{array}\right],
\end{aligned}
$$

$$
\begin{aligned}
& \mathscr{R}=\left[\begin{array}{cc}
0.0016 & -0.0004 \\
-0.0004 & 0.0020
\end{array}\right], \\
& \mathscr{T}=10^{-3}\left[\begin{array}{cc}
-0.5221 & 0.1113 \\
0.1113 & -0.6436
\end{array}\right], \\
& \mathscr{Z}=10^{-6}\left[\begin{array}{ll}
0.7517 & 0.9557 \\
0.9557 & 0.1196
\end{array}\right], \\
& \mathscr{Z}_{1}=\left[\begin{array}{ll}
0.1156 & -0.0845 \\
-0.0845 & 0.1132
\end{array}\right],
\end{aligned}
$$$$
\mathcal{S}_{11}=10^{-4}\left[\begin{array}{cc}
-0.1043 & 0.0205 \\
0.0205 & -0.1511
\end{array}\right] \text {, }
$$$$
\mathcal{S}_{21}=10^{-6}\left[\begin{array}{cc}
-0.2608 & -0.0113 \\
-0.0113 & 0.1344
\end{array}\right] \text {, }
$$$$
\mathcal{S}_{31}=10^{-4}\left[\begin{array}{cc}
-0.1080 & 0.0695 \\
0.0695 & -0.1348
\end{array}\right] \text {, }
$$$$
\mathcal{S}_{41}=10^{-5}\left[\begin{array}{cc}
0.0974 & 0.0430 \\
0.0430 & -0.1378
\end{array}\right] \text {, }
$$$$
\mathcal{S}_{12}=10^{-2}\left[\begin{array}{ll}
-0.1749 & -0.0102 \\
-0.0102 & -0.2199
\end{array}\right] \text {, }
$$$$
\mathcal{S}_{22}=10^{-6}\left[\begin{array}{cc}
-0.7636 & -0.0203 \\
-0.0203 & 0.4242
\end{array}\right] \text {, }
$$$$
\mathcal{S}_{32}=10^{-4}\left[\begin{array}{cc}
-0.3125 & 0.4207 \\
0.4207 & -0.5928
\end{array}\right] \text {, }
$$$$
\mathcal{S}_{42}=10^{-5}\left[\begin{array}{cc}
0.1445 & -0.1174 \\
-0.1174 & 0.0773
\end{array}\right] \text {, }
$$$$
\rho=6.5704
$$$$
\alpha=2.0583,
$$$$
\beta=9.7893 \text {, }
$$$$
\gamma=0.0037
$$

The maximum allowable upper bounds $\mu$ obtained for different values of $\tau$ are listed in Table 1.

For example simulation, we examine the initial conditions of $x_{i}(t)=[-2,0.15]$ with mode $i=1,2$ depicted in Figure 1, the state response from which we can see that all the components converge to zero. Figures 2 and 3 illustrate the synchronization error of system (25) and 
TABLE 1: Maximum allowable bound for $\mu$ with different values of $\tau$.

\begin{tabular}{cccccccc}
\hline$\tau$ & 0.5 & 0.6 & 0.7 & 0.8 & 0.9 & 0.10 & 1.0 \\
$\mu$ & 1.3456 & 1.2789 & 1.0018 & 0.7356 & 0.6345 & 0.5890 & 0.2359 \\
\hline
\end{tabular}

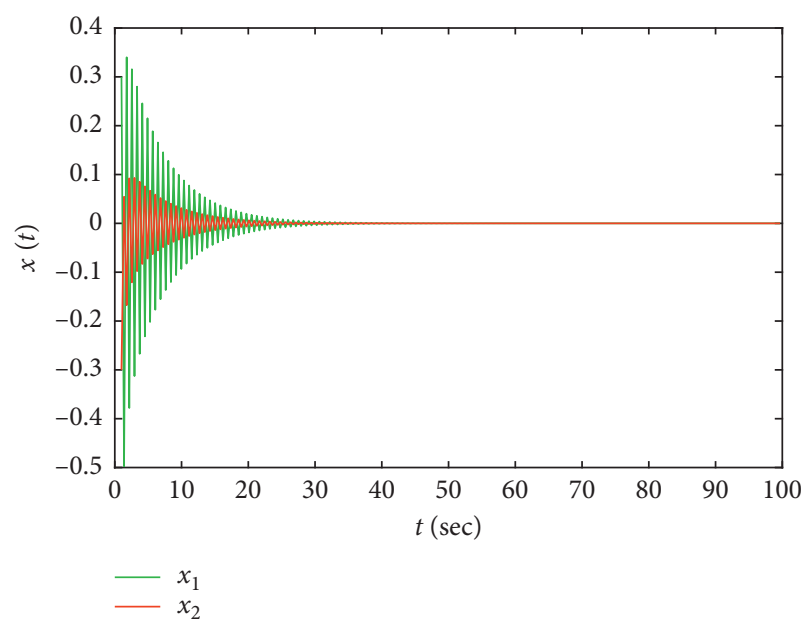

Figure 1: State responses of the system in Example 1.

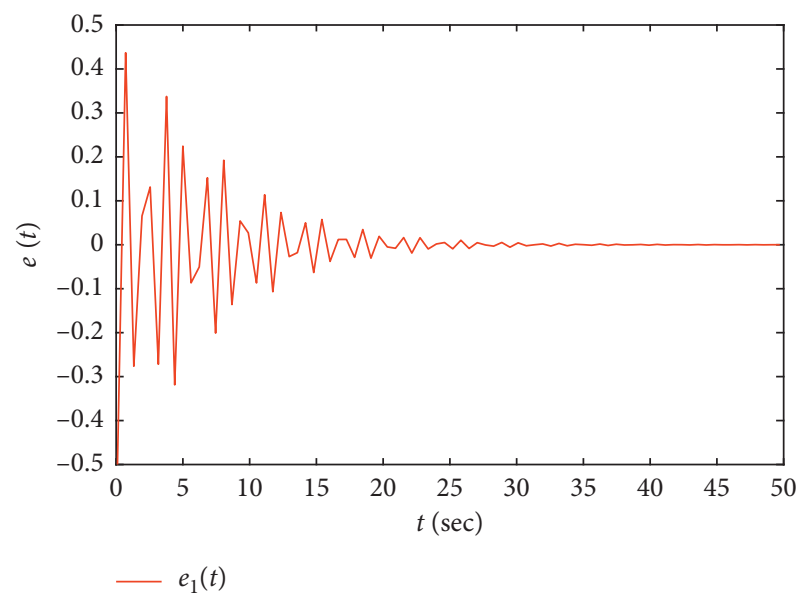

FIGURE 2: Synchronization error of system (25).

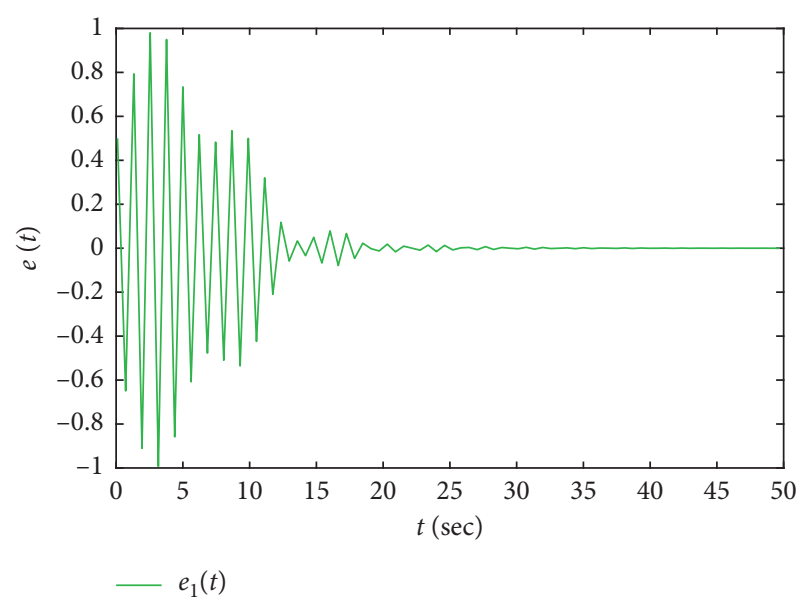

FIgURE 3: Synchronization error of the system in Example 1. consider the networks (9) and (12). State response of the errors $e_{i}(t)$ is illustrated by Figure 4 . Additionally, Figure 5 shows the jumping between models during simulation. From the simulations, one can find that the T-S fuzzy Markovian jumping complex networks are stochastically synchronous.

Example 2. Consider the stochastic complex networks (75) with the following matrix parameters:

$$
\begin{aligned}
& \mathscr{A}_{1}=\left[\begin{array}{cc}
-0.5 & 0.2 \\
0 & 0.95
\end{array}\right], \\
& \mathscr{B}_{1}=\left[\begin{array}{ll}
1 & 0 \\
0 & 1
\end{array}\right], \\
& \mathscr{C}_{1}=\left[\begin{array}{ll}
1 & 0 \\
0 & 1
\end{array}\right],
\end{aligned}
$$

$$
\begin{aligned}
& \mathscr{D}_{1}=\left[\begin{array}{ll}
1 & 0 \\
0 & 1
\end{array}\right], \\
& \mathscr{G}_{11}=\left[\begin{array}{ccc}
-0.2 & 0.1 & 0.1 \\
0.1 & -0.2 & 0.1 \\
0.1 & 0.1 & -0.2
\end{array}\right] \text {, } \\
& \mathscr{G}_{21}=\left[\begin{array}{ccc}
-0.3 & 0.1 & 0.2 \\
0.1 & -0.3 & 0.2 \\
0.1 & 0.2 & -0.3
\end{array}\right] \text {, } \\
& \Gamma_{1}=\Gamma_{1}=\left[\begin{array}{ll}
0.5 & 0 \\
0 & 0.5
\end{array}\right] \text {, } \\
& \mathscr{X}_{1}=\operatorname{disg}\left\{\begin{array}{ll}
0 & 0
\end{array}\right. \text {, } \\
& X_{2}=\operatorname{disg}\{0.20 .75\} \text {, } \\
& \mathscr{Y}_{1}=\operatorname{disg}\{0.20 .1\} \text {, } \\
& \mathscr{Y}_{2}=\operatorname{disg}\{0.10 .1\} \text {, } \\
& \mathscr{H}_{1}=\operatorname{disg}\{0.20 .1\} \text {, } \\
& \mathscr{H}_{2}=\operatorname{disg}\{0.10 .1\} \text {. }
\end{aligned}
$$

Let $\varrho=1, \tau=1.5, \mu=0.5, W=3$. Solve Example 2 using LMIs in Corollary 2. The maximum value of upper bound $\tau$ compared with the results in [67] is listed in Table 2. One can know clearly that the results obtained by Corollary 2 can provide large admissible upper bounds than the stability criteria in [67]. 


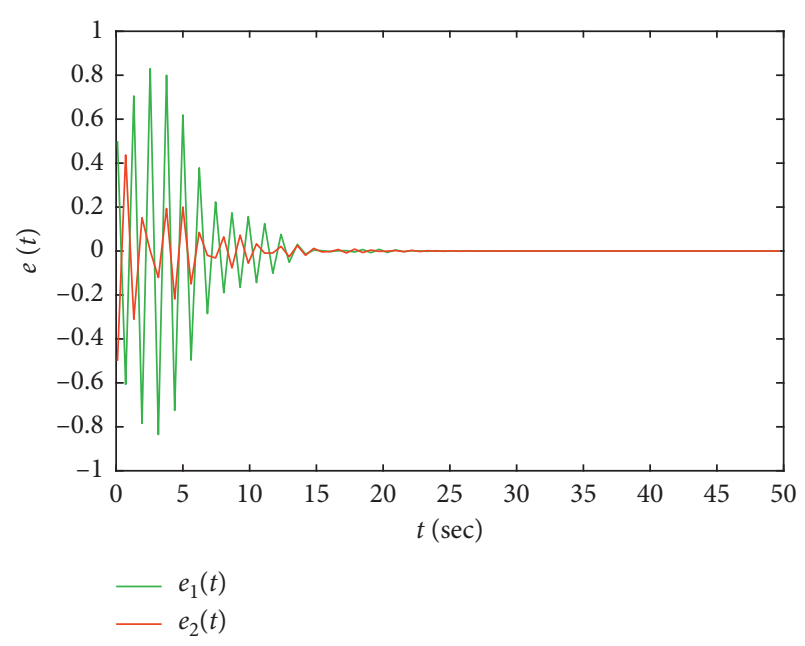

Figure 4: State responses of the error system.

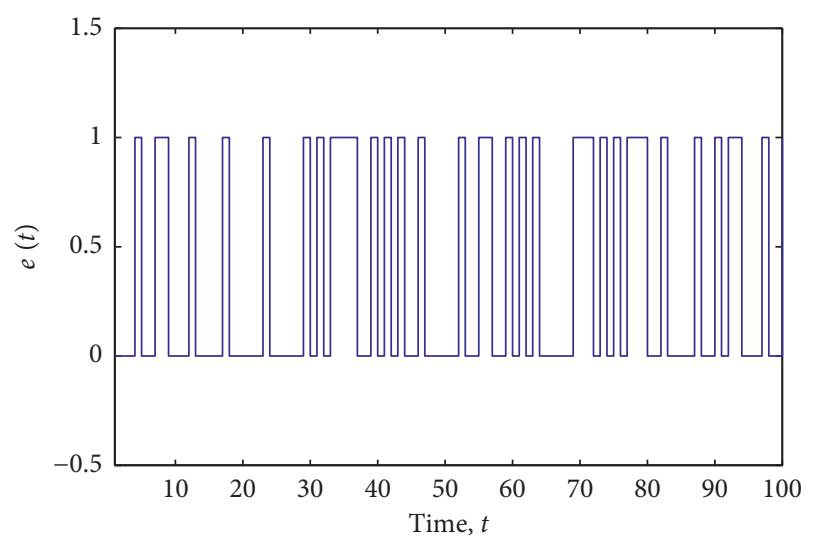

FIGURE 5: Jumping between modes during simulation.

TABLE 2: The corresponding allowable upper bound of time delay $\tau$ for Example 2.

\begin{tabular}{lcc}
\hline Method & {$[67]$} & Corollary 2 \\
Example 2 & 1.5001 & 2.5670 \\
\hline
\end{tabular}

\section{Conclusion}

In this study, we obtained a novel design of a synchronization analysis for T-S fuzzy complex networks' Markovian jumping parameters with stochastic discrete and distributed time-varying delays by means of impulsive control. By using Kronecker product and stochastic examination hypothesis, constructing an appropriate Lyapunov-Krasovskii functional (LKF), and employing Newton-Leibniz formulation, we show that the synchronization problem of drive-response framework is resolvable in terms of linear matrix inequalities (LMIs) which are feasible. At last, the effectiveness of the theoretical results were illustrated with numerical examples and their simulations. In the further examinations, we will look through the networks with generalizability to deal with some other problems on fault tolerant control and stochastic synchronization of Markovian switching for complex dynamical networks.

\section{Data Availability}

No data were used to support this study.

\section{Conflicts of Interest}

The authors declare that they have no conflicts of interest.

\section{References}

[1] D. Ye, X. Yang, and L. Su, "Fault-tolerant synchronization control for complex dynamical networks with semi-Markov jump topology," Applied Mathematics and Computation, vol. 312, pp. 36-48, 2017.

[2] H. Du, P. Shi, and N. Lü, "Function projective synchronization in complex dynamical networks with time delay via hybrid feedback control," Nonlinear Analysis: Real World Applications, vol. 14, no. 2, pp. 1182-1190, 2013.

[3] M. J. Park, O. M. Kwon, J. H. Park, S. M. Lee, and E. J. Cha, "Synchronization criteria of fuzzy complex dynamical networks with interval time-varying delays," Applied Mathematics and Computation, vol. 218, no. 23, pp. 11634-11647, 2012.

[4] Y. Liu, Z. Wang, and X. Liu, "An LMI approach to stability analysis of stochastic high-order Markovian jumping neural networks with mixed time delays," Nonlinear Analysis: Hybrid Systems, vol. 2, no. 1, pp. 110-120, 2008.

[5] Y. Wu, S. Yan, M. Fan, and W. Li, "Stabilization of stochastic coupled systems with Markovian switching via feedback control based on discrete-time state observations," International Journal of Robust and Nonlinear Control, vol. 28, no. 1, pp. 247-265, 2018.

[6] J. Geng, M. Liu, and Y. Zhang, "Stability of a stochastic onepredator-two-prey population model with time delays," Communications in Nonlinear Science and Numerical Simulation, vol. 53, pp. 65-82, 2017.

[7] J. Yu and M. Liu, "Stationary distribution and ergodicity of a stochastic food-chain model with Lévy jumps," Physica A: Statistical Mechanics and Its Applications, vol. 482, pp. 14-28, 2017.

[8] Y. Liu, W. Li, and J. Feng, "Graph-theoretical method to the existence of stationary distribution of stochastic coupled systems," Journal of Dynamics and Differential Equations, vol. 30, no. 2, pp. 667-685, 2018.

[9] Z. Qiao, Q. Guo, H. Sun, Z. Pan, Y. Liu, and W. Xiong, “An interval gas flow analysis in natural gas and electricity coupled networks considering the uncertainty of wind power," Applied Energy, vol. 201, pp. 343-353, 2017.

[10] B. A. Huberman and L. A. Adamic, "Growth dynamics of the world-wide web," Nature, vol. 401, no. 6749, p. 131, 1999.

[11] M. E. J. Newman, "The structure and function of complex networks," SIAM Review, vol. 45, no. 2, pp. 167-256, 2003.

[12] R. Pastor-Satorras and A. Vespignani, "Epidemic spreading in scale-free networks," Physical Review Letters, vol. 86, no. 14, pp. 3200-3203, 2001.

[13] C. W. Wu, "Synchronization in small-word systems," Physical Review Letters, vol. 89, pp. 54-101, 2002.

[14] X. F. Wang and G. Chen, "Synchronization in scale-free dynamical networks: robustness and fragility," IEEE Transactions on Circuits and Systems I, vol. 49, no. 1, pp. 54-62, 2002.

[15] J. Lu, D. W. C. Ho, and J. Cao, "A unified synchronization criterion for impulsive dynamical networks," Automatica, vol. 46, no. 7, pp. 1215-1221, 2010. 
[16] J. Lu, D. Ho, and J. Kurths, "Consensus over directed static networks with arbitrary communication delays," Physics Review E, vol. 80, no. 6, Article ID 066121, 2009.

[17] H. Gao, J. Lam, and G. Chen, "New criteria for synchronization stability of general complex dynamical networks with coupling delays," Physics Letters A, vol. 360, no. 2, pp. 263273, 2006.

[18] C. W. Wu, Synchronization in Complex Networks of Nonlinear Dynamical Systems, World Scientific, Singapore, 2007.

[19] S. Chen, H. Pei, Q. Lai, and H. Yan, "Multitarget tracking control for coupled heterogeneous inertial agents systems based on flocking behavior," IEEE Transactions Systems Man Cybernetics Systems, vol. 49, no. 12, pp. 2605-2611, 2018.

[20] J. Cao, G. Chen, and P. Li, "Global synchronization in an array of delayed neural networks with hybrid coupling," IEEE Transactions Systems Man Cybernetics, Part B, Cybernetics, vol. 38, pp. 488-498, 2008.

[21] M. Chen and W.-h. Chen, "Robust adaptive neural network synchronization controller design for a class of time delay uncertain chaotic systems," Chaos, Solitons \& Fractals, vol. 41, no. 5, pp. 2716-2724, 2009.

[22] J. Cao, Z. Wang, and Y. Sun, "Synchronization in an array of linearly stochastically coupled networks with time delays," Physica A: Statistical Mechanics and Its Applications, vol. 385, no. 2, pp. 718-728, 2007.

[23] N. N. Krasovskii and E. A. Lidskii, "Analysis and design of controllers in systems with random attributes," Automat Remote Control, vol. 22, pp. 1021-1025, 1961.

[24] Q. Zhu and J. Cao, "Stability analysis for stochastic neural networks of neutral type with both Markovian jump parameters and mixed time delays," Neurocomputing, vol. 73, no. 13-15, pp. 2671-2680, 2010.

[25] P. Balasubramanian, G. Nagamani, and R. Rakkiyappan, "Passivity analysis for neural networks of neutral type with Markovian jumping parameters and time delay in the leakage term," Communication Nonlinear Science Numerical Simulation, vol. 16, no. 11, pp. 4422-4437, 2011.

[26] D. Tong, Q. Zhu, W. Zhou, Y. Xu, and J. A. Fang, "Adaptive synchronization for stochastic T-S fuzzy neural networks with time-delay and Markovian jumping parameters," Neurocomputing, vol. 117, pp. 91-97, 2013.

[27] Y. Ou, P. Shi, and H. Liu, "A mode-dependent stability criterion for delayed discrete-time stochastic neural networks with Markovian jumping parameters," Neurocomputing, vol. 73, no. 7-9, pp. 1491-1500, 2010.

[28] X. Li and S. Song, "Impulsive control for existence, uniqueness, and global stability of periodic solutions of recurrent neural networks with discrete and continuously distributed delays," IEEE Transactions on Neural Networks and Learning Systems, vol. 24, no. 6, pp. 868-77, 2013.

[29] X. Li and S. Song, "Stabilization of delay systems: delay-dependent impulsive control," IEEE Transactions on Automatic Control, vol. 62, no. 1, pp. 406-411, 2017.

[30] X. Li and J. Wu, "Stability of nonlinear differential systems with state-dependent delayed impulses," Automatica, vol. 64, pp. 63-69, 2016.

[31] Y. Zhang, J. Sun, and G. Feng, "Impulsive control of discrete systems with time delay," IEEE Transactions on Automatic Control, vol. 54, pp. 871-875, 2009.

[32] J. d. J. Rubio, "Robust feedback linearization for nonlinear processes control," ISA Transactions, vol. 74, pp. 155-164, 2018.
[33] J. De Jesus Rubio, J. F. Novoa, G. Ochoa et al., "Structure regulator for the perturbations attenuation in a quadrotor," IEEE Access, vol. 7, no. 1, pp. 138244-138252, 2019.

[34] X. Gao, B. Sun, X. Hu, and K. Zhu, "Echo state network for extended state observer and sliding mode control of vehicle drive motor with unknown hysteresis nonlinearity," Mathematical Problems in Engineering, vol. 2020, Article ID 2534038, 13 pages, 2020.

[35] J. De Jesus Rubio, D. I. Martine, V. Garcia et al., "The perturbations estimation in two gas plants," IEEE Access, vol. 8, 2020.

[36] C. A. Ibanez and M. S. Suarez-Castanon, "A trajectory planning based controller to regulate an uncertain $3 \mathrm{D}$ overhead crane system," International Journal of Applied Mathematics and Computer Science, vol. 29, no. 4, pp. 693702, 2019.

[37] H. Wu and J. Sun, " $p$-moment stability of stochastic differential equations with impulsive jump and Markovian switching," Automatica, vol. 42, no. 10, pp. 1753-1759, 2006.

[38] H. K. Lam, H. Li, C. Deters, E. L. Secco, H. A. Wurdemann, and K. Althoefer, "Control design for interval type-2 fuzzy systems under imperfect premise matching," IEEE Transactions on Industrial Electronics, vol. 61, no. 2, pp. 956-968, 2014.

[39] M. Syed Ali, N. Gunasekaran, and Q. Zhu, "State estimation of T-S fuzzy delayed neural networks with Markovian jumping parameters using sampled-data control," Fuzzy Sets and Systems, vol. 306, pp. 87-104, 2017.

[40] R. Tang, X. Yang, X. Wan, Y. Zou, Z. Cheng, and H. M. Fardoun, "Finite-time synchronization of nonidentical BAM discontinuous fuzzy neural networks with delays and impulsive effects via non-chattering quantized control," Communications in Nonlinear Science and Numerical Simulation, vol. 78, Article ID 104893, 2019.

[41] N. Gunasekaran, R. Saravanakumar, Y. H. Joo, H. S. Kim, and H. S. Kim, "Finite-time synchronization of sampled-data T-S fuzzy complex dynamical networks subject to average dwelltime approach," Fuzzy Sets and Systems, vol. 374, pp. 40-59, 2019.

[42] O. M. Kwon, M. J. Park, S. M. Lee, and J. H. Park, “Augmented Lyapunov-Krasovskii functional approaches to robust stability criteria for uncertain Takagi-Sugeno fuzzy systems with time-varying delays," Fuzzy Sets and Systems, vol. 201, pp. 1-19, 2012.

[43] K. Tanaka and H. O. Wang, Fuzzy Control Systems Design and Analysis: A Linear Matrix Inequality Approach, Wiley, New York, NY, USA, 2001.

[44] M. Chadli, H. R. Karimi, and P. Shi, "On stability and stabilization of singular uncertain Takagi-Sugeno fuzzy systems," Journal of The Franklin Institute, vol. 351, no. 3, pp. 14531463, 2014.

[45] Y. Liu, B.-Z. Guo, and J. H. Park, "Non-fragileHofiltering for delayed Takagi-Sugeno fuzzy systems with randomly occurring gain variations," Fuzzy Sets and Systems, vol. 316, pp. 99-116, 2017.

[46] T. Takagi and M. Sugeno, "Fuzzy identification of systems and its applications to modeling and control," IEEE Transactions on Systems, Man, and Cybernetics, vol. SMC-15, no. 1, pp. 116-132, 1985.

[47] P. Balasubramaniam, M. Syed Ali, and S. Arik, "Global asymptotic stability of stochastic fuzzy cellular neural networks with multiple time-varying delays," Expert Systems with Applications, vol. 37, no. 12, pp. 7737-7744, 2010. 
[48] J. An and G. Wen, "Improved stability criteria for timevarying delayed T-S fuzzy systems via delay partitioning approach," Fuzzy Sets and Systems, vol. 185, no. 1, pp. 83-94, 2011.

[49] S. Tong, Y. Li, Y. Li, and Y. Liu, "Observer-based adaptive fuzzy back stepping control for a class of stochastic nonlinear strict-feedback systems," IEEE Transactions on Systems, Man, and Cybernetics, Part B, vol. 41, pp. 1693-1704, 2011.

[50] S. Tong, T. Wang, Y. Li, and B. Chen, "A combined backstepping and stochastic small-gain approach to robust adaptive fuzzy output feedback control," IEEE Transactions on Fuzzy Systems, vol. 21, no. 2, pp. 314-327, 2013.

[51] Y. Liu, S. Zhao, and J. Lu, "A new fuzzy impulsive control of chaotic systems based on T-S fuzzy model," IEEE Transactions on Fuzzy Systems, vol. 19, no. 2, pp. 393-398, 2011.

[52] Z. Li, Fuzzy Chaotic Systems: Modeling, Control, and Applications (Studies in Fuzziness and Soft Computing), SpringerVerlag New York, Inc., New York, NY, USA, 2006.

[53] X. Mao, Stochastic Differential Equations and Their Applications, Horwood Publishing, Chichester, UK, 1997.

[54] Z.-G. Wu, P. Shi, H. Su, and J. Chu, "Stochastic synchronization of markovian jump neural networks with time-varying delay using sampled data," IEEE Transactions on Cybernetics, vol. 43, no. 6, pp. 1796-1806, 2013.

[55] M. Syed Ali, J. Yogambigai, and O. M. Kwon, "Finite-time robust passive control for a class of switched reaction-diffusion stochastic complex dynamical networks with coupling delays and impulsive control," International Journal of Systems Science, vol. 49, no. 4, pp. 718-735, 2018.

[56] X. Mao, "Exponential stability of stochastic delay interval systems with Markovian switching," IEEE Transactions on Automatic Control, vol. 47, pp. 1604-1612, 2002.

[57] Y. Liu, Z. Wang, and X. Liu, "Synchronization and state estimation fordiscrete-time complex networks with distributed delays," IEEE Transactions on Systems, Man and Cybernetics, vol. 38, no. 5, pp. 1314-1325, 2008.

[58] X. Song, S. Xu, and H. Shen, "Robust Ho control for uncertain fuzzy systems with distributed delays via output feedback controllers," Information Sciences, vol. 178, no. 22, pp. 4341-4356, 2008.

[59] Y. Tang, R. Qiu, J.-a. Fang, Q. Miao, and M. Xia, “Adaptive lag synchronization in unknown stochastic chaotic neural networks with discrete and distributed time-varying delays," Physics Letters A, vol. 372, no. 24, pp. 4425-4433, 2008.

[60] B. Huang, H. Zhang, D. Gong, and J. Wang, "Synchronization analysis for static neural networks with hybrid couplings and time delays," Neurocomputing, vol. 148, pp. 288-293, 2015.

[61] Y. Tang, J. Fang, and M. Xia, "Synchronization of TakagiSugeno fuzzy stochastic complex networks with mixed delays," in Proceedings of the 2009 Second International Conference on Intelligent Computation Technology and Automation, vol. 2, pp. 810-813, October 2009, Changsha, China.

[62] Y. Tang, J.-a. Fang, M. Xia, and X. Gu, "Synchronization of Takagi-Sugeno fuzzy stochastic discrete-time complex networks with mixed time-varying delays," Applied Mathematical Modelling, vol. 34, no. 4, pp. 843-855, 2010.

[63] D. Gong, H. Zhang, Z. Wang, and J. Liu, "Synchronization analysis for complex networks with coupling delay based on T-S fuzzy theory," Applied Mathematical Modelling, vol. 36, no. 12, pp. 6215-6224, 2012.

[64] D. Yue, E. G. Tian, Y. J. Zhang, and C. Peng, "Delay-distribution-dependent stability and stabilization of T-S fuzzy systems with probabilistic interval delay," IEEE Transactions on Systems, Man, and Cybernetics B, vol. 39, pp. 503-516, 2009.

[65] C. Huang, D. W. C. Ho, J. Lu, and J. Kurths, "Pinning synchronization in T-S fuzzy complex networks with partial and discrete-time couplings," IEEE Transactions on Fuzzy Systems, vol. 23, no. 4, pp. 1274-1285, 2015.

[66] Y. Wu, R. Lu, P. Shi, H. Su, and Z.-G. Wu, "Sampled-data synchronization of complex networks with partial couplings and T-S fuzzy nodes," IEEE Transactions on Fuzzy Systems, vol. 26, no. 2, pp. 782-793, 2018.

[67] M. Syed Ali, M. Usha, J. Cao, and G. Lu, "Synchronization analysis for stochastic T-S fuzzy complex networks with coupling delay," International Journal of Systems Science, vol. 50, no. 3, pp. 585-598, 2019. 